\title{
On the local in time well-posedness of an elliptic-parabolic ferroelectric phase-field model
}

\author{
Yongming Luo *†
}

\begin{abstract}
We consider a state-of-the-art ferroelectric phase-field model arising from the engineering area in recent years, which is mathematically formulated as a coupled elliptic-parabolic differential system. We utilize the maximal parabolic regularity theory to show the local in time wellposedness of the ferroelectric problem in both 2D and 3D spaces, which is sharp in the sense that the local solution is unique and a blow-up criterion is present. The well-posedness result will firstly be proved under some general assumptions. Afterwards we give sufficient geometric and regularity conditions which will guarantee the fulfillment of the imposed assumptions.
\end{abstract}

\section{Introduction}

In this paper, we study a state-of-the-art ferroelectric phase-field model [41, 40] arising from the engineering area in recent years. To formulate the problem, we list the corresponding physical quantities in Table 1 below.

Given a bounded domain $\Omega \subset \mathbb{R}^{d}$ and a time slot $(0, T)$ with $T \in(0, \infty)$, the following constitutive laws are imposed for $\boldsymbol{\sigma}$ and $\mathbf{D}$ :

$$
\begin{aligned}
\boldsymbol{\sigma} & =\mathbb{C}(\mathbf{P})\left(\varepsilon(\mathbf{u})-\varepsilon^{0}(\mathbf{P})\right)+\mathbf{e}(\mathbf{P})^{T} \nabla \phi & \text { in }(0, T) \times \Omega, \\
\mathbf{D} & =\mathbf{e}(\mathbf{P})\left(\boldsymbol{\varepsilon}(\mathbf{u})-\boldsymbol{\varepsilon}^{0}(\mathbf{P})\right)-\boldsymbol{\epsilon}(\mathbf{P}) \nabla \phi+\mathbf{P} & \text { in }(0, T) \times \Omega .
\end{aligned}
$$

We aim to find a solution $(\mathbf{u}, \phi, \mathbf{P})$ of the Cauchy problem

$$
\begin{aligned}
-\operatorname{div} \boldsymbol{\sigma}=\mathrm{f}_{\boldsymbol{\sigma}} & \text { in }(0, T) \times \Omega, \\
-\operatorname{div} \mathbf{D}=\mathrm{f}_{\mathbf{D}} & \text { in }(0, T) \times \Omega, \\
\mathbf{P}^{\prime}=\Delta \mathbf{P}-D_{\mathbf{P}} H(\varepsilon(\mathbf{u}), \nabla \phi, \mathbf{P})-D_{\mathbf{P}} \omega(\mathbf{P})+\mathrm{f}_{\mathbf{P}} & \text { in }(0, T) \times \Omega
\end{aligned}
$$

with the boundary conditions

$$
\begin{array}{ll}
\mathbf{u}=0 & \text { on }(0, T) \times \Gamma_{\mathbf{u}}, \\
\phi=0 & \text { on }(0, T) \times \Gamma_{\phi}, \\
\mathbf{P}=0 & \text { on }(0, T) \times \Gamma_{\mathbf{P}},
\end{array}
$$

*Institut für Wissenschaftliches Rechnen, Technische Universität Dresden, Germany 'Email: yongming.luo@tu-dresden.de 
Table 1: List of variables

\begin{tabular}{|r|c|}
\hline Mechanical displacement & $\mathbf{u}:[0, T] \times \Omega \rightarrow \mathbb{R}^{d}$ \\
Infinitesimal small strain tensor & $\boldsymbol{\varepsilon}(\mathbf{u}):=\left(\nabla \mathbf{u}+\nabla \mathbf{u}^{T}\right) / 2$ \\
Electric potential & $\phi:[0, T] \times \Omega \rightarrow \mathbb{R}^{\circ}$ \\
Spontaneous polarization & $\mathbf{P}:[0, T] \times \Omega \rightarrow \mathbb{R}^{d}$ \\
Cauchy stress tensor & $\boldsymbol{\sigma}:[0, T] \times \Omega \rightarrow \operatorname{Lin}_{\text {sym }}\left(\mathbb{R}^{d}\right)$ \\
Dielectric displacement & $\mathbf{D}:[0, T] \times \Omega \rightarrow \mathbb{R}^{d}$ \\
Elastic stiffness tensor & $\mathbb{C}: \mathbb{R}^{d} \rightarrow \operatorname{Lin}_{\mathrm{sym}}\left(\mathbb{R}^{d \times d}\right)$ with $\mathbb{C}_{i j}^{k l}=\mathbb{C}_{j i}^{l k}$ \\
Symmetric plastic strain tensor & $\boldsymbol{\varepsilon}^{0}: \mathbb{R}^{d} \rightarrow \operatorname{Lin}$ sym $\left(\mathbb{R}^{d}\right)$ \\
Coupling effect tensor & $\mathbf{e}: \mathbb{R}^{d} \rightarrow \operatorname{Lin}\left(\mathbb{R}^{d \times d}, \mathbb{R}^{d}\right)$ \\
Symmetric dielectric matrix & $\boldsymbol{\epsilon}: \mathbb{R}^{d} \rightarrow \operatorname{Lin}_{\text {sym }}\left(\mathbb{R}^{d}\right)$ \\
Separation energy density & $\omega: \mathbb{R}^{d} \rightarrow \mathbb{R}$
\end{tabular}

$$
\begin{aligned}
\boldsymbol{\sigma} \mathbf{n}=\mathrm{t}_{\boldsymbol{\sigma}} & \text { on }(0, T) \times \Gamma_{\mathbf{u}}^{\mathrm{N}} \\
\mathbf{D} \cdot \mathbf{n}=\mathrm{t}_{\mathbf{D}} & \text { on }(0, T) \times \Gamma_{\phi}^{\mathrm{N}} \\
\partial \mathbf{P} / \partial \mathbf{n}=\mathrm{t}_{\mathbf{P}} & \text { on }(0, T) \times \Gamma_{\mathbf{P}}^{\mathrm{N}}
\end{aligned}
$$

and the initial condition

$$
\mathbf{P}(0, x)=\mathbf{P}_{0}(x) \quad \text { in } \Omega,
$$

where $\Gamma_{\mathbf{u}} \dot{\cup} \Gamma_{\mathbf{u}}^{\mathrm{N}}=\Gamma_{\phi} \dot{\cup} \Gamma_{\phi}^{\mathrm{N}}=\Gamma_{\mathbf{P}} \dot{\cup} \Gamma_{\mathbf{P}}^{\mathrm{N}}=\partial \Omega 1, \mathbf{n}$ is the outer normal vector, $\mathrm{f}_{\boldsymbol{\sigma}}, \mathrm{f}_{\mathbf{D}}, \mathrm{f}_{\mathbf{P}}$ are the body force densities, $\mathrm{t}_{\boldsymbol{\sigma}}, \mathrm{t}_{\mathbf{D}}, \mathrm{t}_{\mathbf{P}}$ are the boundary flux densities and $H$ is the bulk energy density defined by

$$
H(\varepsilon, \nabla \phi, \mathbf{P})=\frac{1}{2} \mathbb{B}(\mathbf{P})\left(\begin{array}{c}
\varepsilon-\varepsilon^{0}(\mathbf{P}) \\
-\nabla \phi
\end{array}\right):\left(\begin{array}{c}
\varepsilon-\varepsilon^{0}(\mathbf{P}) \\
\nabla \phi
\end{array}\right)+\nabla \phi \cdot \mathbf{P}
$$

with

$$
\mathbb{B}(\mathbf{P})=\left(\begin{array}{cc}
\mathbb{C}(\mathbf{P}) & \mathbf{e}(\mathbf{P})^{T} \\
-\mathbf{e}(\mathbf{P}) & \boldsymbol{\epsilon}(\mathbf{P})
\end{array}\right)
$$

A schematic diagram for the boundary partition $\partial \Omega=\Gamma_{\mathbf{u}} \dot{\cup} \Gamma_{\mathbf{u}}^{\mathrm{N}}$ is depicted in Fig. 1 below.

The study of ferroelectric materials has a longstanding history since its very first discovery by Valasek 42 in the 20th century. A crucial property of the ferroelectric materials is their ability of transforming an electric signal into mechanical deformation, and vice versa. Such property, which is known as the piezoelectricity, displays the linear counterpart of a ferroelectric model. However, when the ferroelectric materials are cooled down below the Curie temperature, they enter a nonlinear regime and their dynamics are described by a nonlinear evolutionary law. Such phase transition phenomenon also changes the dipole moments between the electric charges within the single crystals, hence

\footnotetext{
${ }^{1}$ Heuristically, $\Gamma_{\mathbf{u}}, \Gamma_{\phi}, \Gamma_{\mathbf{P}}$ and $\Gamma_{\mathbf{u}}^{\mathrm{N}}, \Gamma_{\phi}^{\mathrm{N}}, \Gamma_{\mathbf{P}}^{\mathrm{N}}$ denote the Dirichlet and Neumann boundary portions respectively.
} 


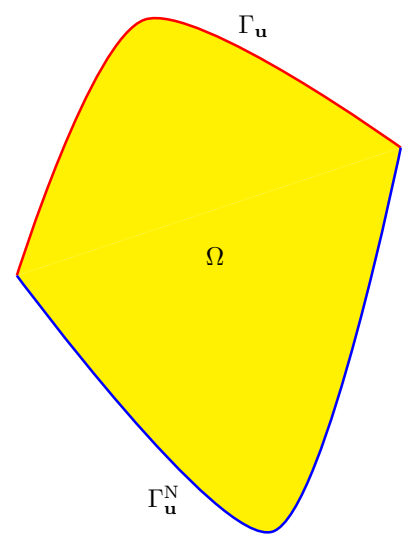

Figure 1: An illustration for the boundary partition $\partial \Omega=\Gamma_{\mathbf{u}} \cup \Gamma_{\mathbf{u}}^{\mathrm{N}}$. The red part and blue part are the Dirichlet and Neumann boundary portions corresponding to the variable $\mathbf{u}$ respectively.

also leads to the spontaneous polarization. Moreover, the spontaneous polarization is aligned along or opposite to the axes of the single tetrahedral crystals, therefore $90^{\circ}$ or $180^{\circ}$ domain walls are also obtained. Each domain wall can thus be seen as a phase-field parameter, and this important feature enables us to study the ferroelectric model using the phase-field theory. The above mentioned properties particularly reveal the suitability of ferroelectric materials for applications involving memory effects, such as sensors, actuators, capacitors etc. For a more comprehensive introduction on ferroelectric materials, we refer to $45,8,17,20,23$ and the references therein. We also refer the readers to [5, 42, 43, 30, 22, 35, 40, for recent progress on the modeling and numerical simulations of the ferroelectric model.

In contrary to the diverse results for ferroelectrics given in the engineering field, a rigorous mathematical qualitative or quantitative analysis for the ferroelectric phase-field model can barely be found in the literature. The first remarkable result concerning the well-posedness of the ferroelectric phase-field model was given by Mielke and Timofte [37. Therein, the ferroelectric problem is considered on a bounded domain $\Omega \subset \mathbb{R}^{d}$ for a time slot $[0, T]$ and formulated in terms of an abstract Helmholtz free energy $\mathcal{H}$ and a non-negative convex dissipation functional $\Psi$. The key assumption made in [37] is the rateindependence of the dissipation functional $\Psi$, i.e.

$$
\forall r \geq 0 \forall \mathbf{P} \in \mathbf{X}_{3}: \Psi(r \mathbf{P})=r \Psi(\mathbf{P}),
$$

where $\mathbf{X}_{3}$ is some suitable underlying function space for $\mathbf{P}$. Relying on certain generalized De Giorgi energy-dissipation principles for rate-independent systems (see for instance [36]), Mielke and Timofte were able to show the existence of a so called energetic solution for rate-independent ferroelectric system. Similar models are considered in [28] and 38, where the conditions on $\mathcal{H}$ are different to the ones in [37] (more precisely, there is no gradient energy term $\frac{1}{2}\|\mathbf{P}\|_{L^{2}(\Omega)}^{2}$ contained in $\mathcal{H}$, and the evolutionary law for $\mathbf{P}$ reduces to an ODE) but the dissipation functionals $\Psi$ remain rate-independent. Recently, different solution 
schemes such as BV-solutions and vanishing viscosity solutions etc. have been successively applied for rate-independent ferroelectric phase-field models. We refer to [25, 29] for further details.

In general, however, ferroelectric materials display rate-dependence on the spontaneous polarization, and the system can be assumed rate-independent only when the external loadings possess a relatively much lower frequency compared to the internal dipoles. In this paper, we will study the rate-dependent ferroelectric model introduced in [41, 40, which is considered as one of the state-of-the-art models in the current research for ferroelectric materials. We focus on the 2D and 3D cases, being the general scenarios under consideration in practical applications. To the author's best knowledge, this is the first rigorous well-posedness result for rate-dependent ferroelectric phase-field models with purely quadratic dissipation growth. A number of interesting features are also displayed by the model:

- The spontaneous polarization $\mathbf{P}$ admits boundary conditions of mixed type2, where $\mathbf{P}$ in the models [37, 25] with pure Neumann boundary conditions are considered.

- The material parameters $\mathbb{C}, \varepsilon^{0}, \mathbf{e}, \boldsymbol{\epsilon}$ are assumed to be functions of $\mathbf{P}$, which are usually in literature merely assumed to be (fully or partially) constants [28, 41] or depend on the spatial variable $x \in \Omega$ [25].

- When the variable $(\mathbf{u}, \phi)$ possesses pure Dirichlet boundary conditions, then no uniform ellipticity and boundedness conditions are imposed on the material parameters, see Theorem 2.6 below.

- The well-posedness result is sharp, in the sense that the local solution is unique and a blow-up criterion is present, see Definition 2.1 below.

We briefly summarize the idea for proving the local well-posedness of the model problem. The proof follows the same lines of 34]: by multiplying a test function and using partial integration, the balance law (1.3) is reformulated to the following coupled elliptic-parabolic system:

$$
\begin{gathered}
-\operatorname{Div}\left(\mathbb{B}(\mathbf{P}(t))\left(\begin{array}{c}
\varepsilon(\mathbf{u}(t)) \\
\nabla \phi(t)
\end{array}\right)\right)=\ell(t, \mathbf{P}(t)) \quad \text { in } \mathbb{W}_{p}^{*}, \\
\mathbf{P}^{\prime}(t)=\Delta \mathbf{P}(t)+F(t, \boldsymbol{\varepsilon}(\mathbf{u}(t)), \nabla \phi(t), \mathbf{P}(t)) \quad \text { in }\left(W_{\Gamma_{\mathbf{P}}}^{-1, p}(\Omega)\right)^{d},
\end{gathered}
$$

where the space $\mathbb{W}_{p}^{*}$ is defined by (3.2). Using Lax-Milgram we infer that $(\mathbf{u}(t), \phi(t))$ as well as the nonlinear functional $F(t, \varepsilon(\mathbf{u}(t)), \nabla \phi(t), \mathbf{P}(t))$ are uniquely determined by the given pair $(t, \mathbf{P}(t))$. Hence $F(t, \boldsymbol{\varepsilon}(\mathbf{u}(t)), \nabla \phi(t), \mathbf{P}(t))$ can be rewritten to the form $S(t, \mathbf{P}(t))$ and (1.8) equivalently reduces to a semilinear parabolic equation

$$
\mathbf{P}^{\prime}(t)=\Delta \mathbf{P}(t)+S(t, \mathbf{P}(t)) .
$$

We then exploit the existence and uniqueness result given by Clément and Li [7] to obtain the local well-posedness of the semilinear parabolic problem (1.9). It is worth noting that the key point for the proof is that the solution $(\mathbf{u}, \phi)$ of (1.8a)

${ }^{2}$ More precisely, $\mathbf{P}$ satisfies (1.4c) and (1.4f) with $\partial \Omega=\Gamma_{\mathbf{P}} \cup \dot{\cup} \Gamma_{\mathbf{P}}^{\mathrm{N}}$. 
is of class $W^{1, p}$ for some $p>d$ (see (4.22) below). This property will firstly be imposed as an assumption (Assumption (A3)) for the local well-posedness result. We then give sufficient geometric and regularity conditions which will guarantee the validity of Assumption (A3). For details, we refer to Section 2

\subsection{Outline of the paper}

The rest of the paper is organized as follows: In Section 2 we state the precise statements of the main results; In Section 3 we list the notation and definitions which will be used throughout this paper; finally, Section 4 is devoted to the proof of Theorem 2.3 and Section 5 to the proofs of Theorems 2.4 2.6 and 2.10.

\section{Assumptions and main results}

In this section we state the main results of the paper. For the corresponding notation and definitions, we refer to Section 3. We firstly define the precise solution concept for the Cauchy problem (1.3) to (1.5):

Definiton 2.1. Let $p \in(d, \infty)$ and $r \in\left(\frac{2 p}{p-d}, \infty\right)$. We say that $(\mathbf{u}, \phi, \mathbf{P})$ with $\mathbf{P}(0, x)=\mathbf{P}_{0}(x)$ is a solution of the Cauchy problem (1.3) to (1.5) on the maximal time interval $(0, \hat{T})$ with $\hat{T} \in(0, T]$ if for each $S \in(0, \hat{T}),(\mathbf{u}, \phi, \mathbf{P})$ satisfies the equations

$$
\begin{gathered}
-\operatorname{Div}\left(\mathbb{B}(\mathbf{P}(t))\left(\begin{array}{c}
\varepsilon(\mathbf{u}(t)) \\
\nabla \phi(t)
\end{array}\right)\right)=\ell(t, \mathbf{P}(t)) \quad \text { in } \mathbb{W}_{p}^{*}, \\
\mathbf{P}^{\prime}(t)=\Delta \mathbf{P}(t)+F(t, \varepsilon(\mathbf{u}(t)), \nabla \phi(t), \mathbf{P}(t)) \quad \text { in }\left(W_{\Gamma_{\mathbf{P}}}^{-1, p}(\Omega)\right)^{d}
\end{gathered}
$$

for a.a. $t \in(0, S)$ and possesses the regularity

$$
\begin{gathered}
(\mathbf{u}, \phi) \in L^{2 r}\left(0, S ; \mathbb{W}_{p}\right), \\
\mathbf{P} \in W^{1, r}\left(0, S ;\left(W_{\Gamma_{\mathbf{P}}}^{-1, p}(\Omega)\right)^{d}\right) \cap L^{r}\left(0, S ;\left(W_{\Gamma_{\mathbf{P}}}^{1, p}(\Omega)\right)^{d}\right) .
\end{gathered}
$$

Here, the spaces $\mathbb{W}_{p}$ and $\mathbb{W}_{p}^{*}$ are defined by

$$
\begin{aligned}
& \mathbb{W}_{p}:=\left(W_{\Gamma_{\mathbf{u}}}^{1, p}(\Omega)\right)^{d} \times W_{\Gamma_{\phi}}^{1, p}(\Omega), \\
& \mathbb{W}_{p}^{*}:=\left(W_{\Gamma_{\mathbf{u}}}^{-1, p}(\Omega)\right)^{d} \times W_{\Gamma_{\phi}}^{-1, p}(\Omega) .
\end{aligned}
$$

Moreover, the maximality of $\hat{T}$ is understood as follows:

(a) Either (2.3) does not hold for $S=\hat{T}$, or

(b) $\hat{T}=T$ and (2.3) also holds for $S=T$.

In the cases (a) and (b), the solution is said to be a finite time blow-up and a global solution respectively. Finally, the tensor $\mathbb{B}(\mathbf{P})$ is defined by (1.7) and the functionals $\ell(t, \mathbf{P}), F(t, \varepsilon, \nabla \phi, \mathbf{P})$ are defined by

$$
\ell(t, \mathbf{P}):=\ell_{1}(\mathbf{P})+\ell_{2}(t)
$$




$$
\begin{aligned}
F(t, \boldsymbol{\varepsilon}, \nabla \phi, \mathbf{P}): & =-D_{\mathbf{P}} H(\varepsilon, \nabla \phi, \mathbf{P})-D_{\mathbf{P}} \omega(\mathbf{P})+\ell_{3}(t) \\
\ell_{1}(\mathbf{P})[\mathbf{u}, \phi]:= & \left(\begin{array}{c}
-\operatorname{Div}_{\mathbf{u}}\left(\mathbb{C}(\mathbf{P}) \varepsilon^{0}(\mathbf{P})\right) \\
\operatorname{Div}_{\phi}\left(\mathbf{e}(\mathbf{P}) \varepsilon^{0}(\mathbf{P})-\mathbf{P}\right)
\end{array}\right)[\mathbf{u}, \phi] \\
& =\int_{\Omega} \mathbb{C}(\mathbf{P}) \varepsilon^{0}(\mathbf{P}): \boldsymbol{\varepsilon}(\mathbf{u})-\left(\mathbf{e}(\mathbf{P}) \varepsilon^{0}(\mathbf{P})-\mathbf{P}\right) \cdot \nabla \phi d x \\
\ell_{2}(t)[\mathbf{u}, \phi]:= & \int_{\Omega} \mathrm{f}_{\boldsymbol{\sigma}}(t, x) \cdot \mathbf{u}(x)-\mathrm{f}_{\mathbf{D}}(t, x) \phi(x) d x+\int_{\Gamma_{\mathbf{u}}^{\mathrm{N}}} \mathrm{t}_{\boldsymbol{\sigma}}(t, x) \cdot \mathbf{u}(x) d S \\
& -\int_{\Gamma_{\phi}^{\mathrm{N}}} \mathrm{t}_{\mathbf{D}}(t, x) \phi(x) d S, \\
\ell_{3}(t)[\mathbf{P}]:= & \int_{\Omega} \mathrm{f}_{\mathbf{P}}(t, x) \cdot \mathbf{P}(x) d x+\int_{\Gamma_{\mathbf{P}}^{\mathrm{N}}} \mathrm{t}_{\mathbf{P}}(t, x) \cdot \mathbf{P}(x) d S
\end{aligned}
$$

for a test function $(\mathbf{u}, \phi, \mathbf{P}) \in \mathbb{W}_{p^{\prime}} \times\left(W_{\Gamma_{\mathbf{P}}}^{1, p^{\prime}}(\Omega)\right)^{d}$.

\subsection{Assumptions}

We impose the following assumptions for the well-posedness result. In particular, the somehow indirectly formulated Assumption (A3) plays a key role for establishing the maximal parabolic regularity of the Laplacian $-\Delta: W_{\Gamma_{\mathbf{P}}}^{1, p}(\Omega) \rightarrow$ $W_{\Gamma_{\mathbf{P}}}^{-1, p}(\Omega)$. In general, it is not an easy task to verify the validity of the abstract Assumption (A3). We will give in Section 5 precise conditions, under which the Assumption (A3) is satisfied.

(A1) $\Omega \subset \mathbb{R}^{d}, d \in\{2,3\}$, is a bounded domain, $\Gamma_{\mathbf{P}}$ is $(d-1)$-set with non-zero surface measure and $\Omega \cup \Gamma_{\mathbf{P}}$ is Gröger-regular (see Definition 3.1).

(A2) $\mathbb{C}, \mathbf{e}, \boldsymbol{\varepsilon}^{0}, \boldsymbol{\epsilon}, \omega$ are $C^{1,1}$-functions on $\mathbb{R}^{d}$.

(A3) There exists some $p \in I_{d}$, where

$$
I_{d}= \begin{cases}(2, \infty), & \text { if } d=2 \\ (3,6], & \text { if } d=3\end{cases}
$$

such that the linear differential operator $L_{\mathbf{P}}$ given by

$$
\operatorname{L}_{\mathbf{P}}(\mathbf{u}, \phi):=-\operatorname{Div}\left(\mathbb{B}(\mathbf{P})\left(\begin{array}{c}
\varepsilon(\mathbf{u}) \\
\nabla \phi
\end{array}\right)\right)
$$

defines a topological isomorphism from $\mathbb{W}_{p}$ to $\mathbb{W}_{p}^{*}$ for all $\mathbf{P}$ in the space $\mathbb{U}$ defined by (3.4).

(A4) There exists some $r \in\left(\frac{2 p}{p-d}, \infty\right)$, where $p$ is the number given by Assumption (A3), such that

$$
\begin{aligned}
& \ell_{2} \in L^{2 r}\left(0, T ; \mathbb{W}_{p}^{*}\right), \\
& \ell_{3} \in L^{r}\left(0, T ;\left(W_{\Gamma_{\mathbf{P}}}^{-1, p}(\Omega)\right)^{d}\right) .
\end{aligned}
$$


Remark 2.2. The restriction $p \in I_{d}$ given in Assumption (A3) comes from the fact that in general it is only expected that the Laplacian $-\Delta: W_{\Gamma_{\mathrm{P}}}^{1, p}(\Omega) \rightarrow$ $W_{\Gamma_{\mathrm{P}}}^{-1, p}(\Omega)$ admits the maximal parabolic regularity for $p \in I_{d}$, see 12 , Thm. $1.6]$.

\subsection{Main results}

Having given the Assumptions (A1) to (A4) we are ready to formulate the local in time well-posedness result:

Theorem 2.3. Let the Assumptions (A1) to (A4) be satisfied. Assume also that

$$
\mathbf{P}_{0} \in\left(\left(W_{\Gamma_{\mathbf{P}}}^{1, p}(\Omega)\right)^{d},\left(W_{\Gamma_{\mathbf{P}}}^{-1, p}(\Omega)\right)^{d}\right)_{\frac{1}{r}, r},
$$

where $(\cdot, \cdot)_{\frac{1}{r}, r}$ denotes the real interpolation. Then the Cauchy problem (1.3) to (1.5) has a unique local solution $(\mathbf{u}, \phi, \mathbf{P})$ in the maximal time interval $(0, \hat{T})$ for some $0<\hat{T} \leq T$ in the sense of Definition 2.1.

In practical applications, the fulfilment of the Assumptions (A1), (A2) and (A4) can be achieved by imposing sufficiently smooth conditions on the underlying domain and the external loadings. The problem hence reduces to finding precise conditions, under which the abstractly formulated Assumption (A3) is satisfied. For 2D case, the Assumption (A3) is an immediate consequence of the elliptic regularity result given by [18. (Theorem 5.2 below). More precisely, we have the following:

Theorem 2.4. Suppose that the following assumptions are satisfied:

(B1) $\Omega \subset \mathbb{R}^{2}$ is a bounded domain, $\Gamma_{\mathbf{u}}, \Gamma_{\phi}$ are closed 1-sets with non-zero surface measure and $\Omega \cup \Gamma_{\mathbf{u}}, \Omega \cup \Gamma_{\phi}$ are Gröger-regular (see Definition 3.1).

(B2) $\mathbb{C}, \mathbf{e}, \varepsilon^{0}, \boldsymbol{\epsilon}$ are essentially bounded on $\mathbb{R}^{2}$.

(B3) There exists some $\alpha>0$ such that for all $\mathbf{P} \in \mathbb{R}^{2}, \varepsilon \in \operatorname{Lin}_{\text {sym }}\left(\mathbb{R}^{2}\right), \mathbf{D} \in \mathbb{R}^{2}$

$$
\begin{gathered}
\mathbb{C}(\mathbf{P}) \varepsilon: \varepsilon \geq \alpha|\varepsilon|^{2}, \\
\boldsymbol{\epsilon}(\mathbf{P}) \mathbf{D} \cdot \mathbf{D} \geq \alpha|\mathbf{D}|^{2} .
\end{gathered}
$$

Then the Assumption (A3) is satisfied.

Remark 2.5. By the setting of Theorem 2.4 a solution $(\mathbf{u}, \phi, \mathbf{P})$ deduced from Theorem 2.3 satisfies

$$
(\mathbf{u}(t), \phi(t), \mathbf{P}(t)) \in\left(W_{\Gamma_{\mathbf{u}}}^{1, p}(\Omega)\right)^{d} \times W_{\Gamma_{\phi}}^{1, p}(\Omega) \times\left(W_{\Gamma_{\mathbf{P}}}^{1, p}(\Omega)\right)^{d}
$$

for a.e. $t \in(0, \hat{T})$. In particular, for a.e. $t \in(0, \hat{T})$ the solution $(\mathbf{u}(t), \phi(t), \mathbf{P}(t))$ has (componentwise) zero trace on the boundary portion $\Gamma_{\mathbf{u}} \times \Gamma_{\phi} \times \Gamma_{\mathbf{P}}$ 
Unfortunately, there is no such elliptic regularity result with $p>3$ by the $3 \mathrm{D}$ case when $(\mathbf{u}, \phi)$ possesses mixed boundary conditions. In fact, to the best knowledge of the author, the sharpest value of $p$ for mixed case is exactly the one given by [18, which is merely expected to be close to two. Nevertheless, if one relaxes the conditions, in the sense that $(\mathbf{u}, \phi)$ satisfies the Dirichlet boundary conditions, then an $L^{p}$-elliptic regularity result with $p>3$ is available (in fact, this is even true for all $p \in(2, \infty))$. Such $L^{p}$-regularity results can either be directly obtained using potential theory, or can also be obtained as a consequence of BMO-estimates for strongly elliptic systems. It is also worth noting that the Dirichlet $L^{p}$-elliptic regularity result is regardless of the dimension, meaning that it is applicable for both $2 \mathrm{D}$ and $3 \mathrm{D}$ cases. By our model, the $L^{p}$-elliptic regularity result is rephrased as follows:

Theorem 2.6. Suppose that the following assumptions are satisfied:

(C1) $\Omega \subset \mathbb{R}^{d}$ with $d \in\{2,3\}$ is an open rectangle (2D) or an open cuboid (3d) or a bounded domain with $C^{1}$-boundary, and $\Gamma_{\mathbf{u}}=\Gamma_{\phi}=\partial \Omega$.

(C2) $\mathbb{C}, \mathbf{e}, \varepsilon^{0}, \boldsymbol{\epsilon}$ are continuous on $\mathbb{R}^{d}$ and there exists some continuous function $\alpha: \mathbb{R}^{d} \rightarrow(0, \infty)$ such that for all $\mathbf{P} \in \mathbb{R}^{d}, \varepsilon \in \operatorname{Lin}_{\mathrm{sym}}\left(\mathbb{R}^{d}\right)$ and $\mathbf{D} \in \mathbb{R}^{d}$

$$
\begin{aligned}
\mathbb{C}(\mathbf{P}) \boldsymbol{\varepsilon}: \boldsymbol{\varepsilon} & \geq \alpha(\mathbf{P})|\varepsilon|^{2}, \\
\boldsymbol{\epsilon}(\mathbf{P}) \mathbf{D} \cdot \mathbf{D} & \geq \alpha(\mathbf{P})|\mathbf{D}|^{2} .
\end{aligned}
$$

Then the Assumption (A3) is satisfied.

The proof of Theorem 2.6 will be a simple application of the regularity results from [1, 10]. We refer to Section 5.2 for details.

Remark 2.7. Compared to Theorem 2.4, we see that in Theorem 2.6 no uniform boundedness and ellipticity conditions are imposed on the material parameters. This is due to the fact that the proof of the elliptic regularity result given in 18 relies on a fixed point iteration, thus uniform upper and lower bounds of the coefficients will be essential for the proof therein. On the other hand, the proof of Theorem 2.6 makes use of local perturbation arguments, we hence only ask for local boundedness and ellipticity.

The particular interest is also devoted to 3D polyhedral domains, being one of the most widely used geometric objects in practical industrial applications. The elliptic regularity theory with symmetric coefficient tensor on polyhedral domains has a long standing history in mathematical research and has been nowadays rather completely developed. We refer the readers to [26, 32] and the references therein for a self-contained study of the elliptic regularity theory on polyhedral domains. The main drawback of the methods given in [26, 32 is that the elliptic regularity on a polyhedral domain is closely related to the spectral properties of the corresponding operator pencil, which is assumed to be self-adjoint, or in other words that the coefficient tensor is required to be symmetric. We recall that the coefficient tensor $\mathbb{B}(\mathbf{P})$ given by $(1.7)$ is in general asymmetric, which prevents us to apply the results from [26, 32 to our model.

Nevertheless, the intrinsic physical principles of the ferroelectric model help us to solve this problem to some extent: a crucial observation from the actual 
ferroelectric model studied in [40] is that the material tensor $\mathbb{B}(\mathbf{P})$ satisfies the condition

$$
\mathbb{B}(0)=\left(\begin{array}{cc}
\lambda \mathbf{I d}_{3} \otimes \mathbf{I d}_{3}+2 \mu \mathbf{E}_{3} & 0 \\
0 & \gamma \mathbf{I} \mathbf{d}_{3}
\end{array}\right),
$$

where $\mathbf{I d}_{3}$ is the identity matrix in $\mathbb{R}^{3 \times 3}, \mathbf{E}_{3}$ is the identity tensor in the space $\operatorname{Lin}_{\text {sym }}\left(\mathbb{R}^{3 \times 3}\right)$ and $\lambda, \mu, \gamma$ are some given positive constants. A differential system with the upper left coefficient tensor in (2.14) is the so called Lamé operator, while the bottom right coefficient tensor in (2.14) corresponds to the Laplace operator. Physically, this phenomenon is to be understood that the piezo system decouples when the spontaneous polarization vanishes. We emphasize here that both Lamé and Laplace operators have symmetric coefficient tensors. The condition (2.14) thus enables us to utilize the theory from [32, combining with a perturbation argument, to derive a reasonable $L^{p}$-elliptic regularity theory for our model. In order to formulate the $L^{p}$-elliptic regularity result for polyhedral domains we will still need the following assumption, which, roughly speaking, ensures that the results from 32 are applicable for any "singular point" on the boundary of the polyhedral domain.

Assumption 2.8. Let $\Omega \subset \mathbb{R}^{3}$ be a bounded polyhedral domain. Denote by $\operatorname{Sing}_{\Omega}^{1}$ the set of singularities of a polyhedral domain $\Omega$, i.e.

$$
\operatorname{Sing}_{\Omega}^{1}:=\{x \in \partial \Omega: x \text { is a vertex or lies on an edge }\} \text {. }
$$

We also define

$$
\operatorname{Sing}_{\Omega}^{2}:=\left\{x \in \partial \Omega: x \text { is a point on some open edge and } \theta_{x}=\pi / 2\right\},
$$

where $\theta_{x}$ is the opening angle of the dihedron $\mathcal{D}_{x}$ defined in Definition 3.5. namely, Sing ${ }_{\Omega}^{2}$ is the set of edge points having $\frac{\pi}{2}$-opening angle;

$\operatorname{Sing}_{\Omega}^{3}:=\{x \in \partial \Omega: x$ is a vertex and

$$
\left.\mathcal{K}_{x} \cap B_{1} \text { is } C^{\infty} \text {-diffeomorph to } \mathcal{K}_{c} \cap B_{1}\right\},
$$

where $\mathcal{K}_{x}$ is the cone defined in Definition 3.5. $B_{1}$ is the unit ball and $\mathcal{K}_{c}$ is the cone formed by the positive $x, y, z$-axis, namely, $\operatorname{Sing}_{\Omega}^{3}$ is the set of cube-cornerlike vertices. Finally, we define

$$
\operatorname{Sing}_{\Omega}:=\operatorname{Sing}_{\Omega}^{1} \backslash\left(\operatorname{Sing}_{\Omega}^{2} \cup \operatorname{Sing}_{\Omega}^{3}\right) .
$$

Then we assume:

- For each vertex $x$ the corresponding cone $\mathcal{K}_{x}$ is a Lipschitz graph with edges, i.e. $\mathcal{K}_{x}$ has the representation $x_{3}>\psi\left(x_{1}, x_{2}\right)$ in Cartesian coordinates with some Lipschitz function $\psi$.

- $\operatorname{Sing}_{\Omega} \subset \Gamma_{\mathbf{P}}$.

Remark 2.9. Here follows an explanation why we need to impose additional regularity assumptions for vertices: One easily sees that every point on an open face or an open edge has an (in general small) neighborhood whose intersection with $\Omega$ has Lipschitz boundary, and the validity of many important features such as Poincaré's inequality and Sobolev embedding etc. is guaranteed. However, a cone need not be a domain with Lipschitz boundary. For a counterexample, we refer to [32, Fig. 9]. 
We now state the last main result of this paper:

Theorem 2.10. Let the Assumption (C2), Assumption 2.8 and (2.14) be satisfied. Let also $\Gamma_{\mathbf{u}}=\Gamma_{\phi}=\partial \Omega$. Then the Assumption (A3) is satisfied.

\section{Notation and definitions}

\subsection{Basic notation}

The symbol $\operatorname{Lin}(A, B)\left(\operatorname{Lin}_{\text {sym }}(A)\right)$ denotes the set of linear (linear symmetric) tensors from $A$ to $B$ (from $A$ to itself) for finite dimensional vector spaces $A$ and $B$. For matrices $M, N \in \mathbb{R}^{m \times n}$, the inner product $M: N$ of $M$ and $N$ is defined by $M: N:=\sum_{i=1}^{m} \sum_{j=1}^{n} M_{i j} N_{i j}$.

For a function $f: \Lambda \subset \mathbb{R}^{m} \rightarrow \mathbb{R}^{n}, D_{x} f(\cdot) \in \mathbb{R}^{n \times m}$ denotes the usual derivative of $f$ in the Euclidean space; for Banach spaces $X, Y$ and a function $f: X \rightarrow Y$, the symbol $D_{x} f(z)[\bar{x}]$ denotes the Gâteaux-differential of $f$ at point $z \in X$ in direction $\bar{x} \in X$. If $\Lambda$ is an open subset of $\mathbb{R}$, then the derivative of $f$ will also be denoted by $f^{\prime}$, which stands for the time derivative of $f$. We also denote by $\nabla f$ the gradient of $f$, i.e. $\nabla f=(D f)^{T}$, where $(D f)^{T}$ is the transpose of the derivative of $f$.

For a differentiable matrix-valued function $v: \Omega \subset \mathbb{R}^{d} \rightarrow \mathbb{R}^{d \times d}$, its divergence $\operatorname{div} v$ is defined as a $d$-dimensional vector with $(\operatorname{div} v)_{i}:=\sum_{j=1}^{d} \partial_{j} v_{i j}$, i.e. the divergence is taken row-wise.

For Banach spaces $X$ and $Y$, we denote by $L(X, Y)$ the space of all functions $f: X \rightarrow Y$ which are linear and continuous. $L(X, Y)$ is a Banach space equipped with the norm $\|f\|_{L(X, Y)}:=\sup _{\|x\|_{X} \leq 1}\|f(x)\|_{Y}$. We denote by $L H(X, Y)$ the subset of $L(X, Y)$ whose elements are additionally bijective.

We will also use the capital letter $C$ to denote a generic large positive number.

\subsection{Geometric settings}

\subsection{1 $l$-set and Gröger-regular set}

In the following, we introduce the geometric concepts l-set and Gröger-regular set, which plays a fundamental role in the proof of the main results:

Definiton 3.1. (i) Let $d \in \mathbb{N}$ and $l \in(0, d]$. We say that a closed set $F \subset \mathbb{R}^{d}$ is an $l$-set, if for all $x \in F$ and $r \in(0,1]$ we have

$$
\mathcal{H}_{l}(F \cap B(x, r)) \sim r^{l},
$$

where $\mathcal{H}_{l}$ denotes the l-dimensional Hausdorff measure.

(ii) A set $W \subset \mathbb{R}^{d}$ is called Gröger-regular, if $W$ is a bounded set and for every $x \in \partial W$, there exist $U_{1}, U_{2} \subset \mathbb{R}^{d}$ and a bi-Lipschitz transformation $\Phi: U_{1} \rightarrow U_{2}$, such that $x \in U_{1}$ and $\Phi\left(U_{1} \cap W\right)$ is one of the following sets:

$$
\begin{aligned}
& \circ M_{1}:=\left\{x \in \mathbb{R}^{d}:\left|x_{i}\right|<1 \forall i=1, \cdots, d \text { and } x_{d}<0\right\}, \\
& \circ M_{2}:=\left\{x \in \mathbb{R}^{d}:\left|x_{i}\right|<1 \forall i=1, \cdots, d \text { and } x_{d} \leq 0\right\}, \\
& \circ M_{3}:=\left\{x \in M_{2}: x_{d}<0 \text { or } x_{1}>0\right\} .
\end{aligned}
$$

Remark 3.2. Here follow several comments on Definition 3.1. 
(i) The concept of $l$-set is also referred to as the $l$-Ahlfors-David-regular set in literature, which was originally proved to be equivalent to the $L^{2}$-boundedness of the Cauchy integral operator appearing in the study of complex geometric analysis. We refer to the papers [2, 9, 31] and the references therein for further details. After its first appearance, the Ahlfors-David-regular condition arises naturally in different research areas of mathematics and plays a fundamental role for many important results. We refer to 21] for some enlightening examples of Ahlfors-David-regularsets. Particularly, the closure of a bounded weakly Lipschitz domain (in the sense of [15, Def. 1.2.1.2]) in $\mathbb{R}^{d}$ is a $d$-set and the boundary of a bounded Lipschitz domain in $\mathbb{R}^{d}$ is a $(d-1)$-set, see for instance [21, Chap. II, Exp. 1] for a proof.

(ii) The Gröger-regular set was originally introduced by Gröger [16 for the purpose of characterizing the geometric profile of domains of mixed type. It turns out that the Gröger-regular condition is not a too restrictive condition and therefore widely applied in different applications. We refer to [19, Sec. 7] for a survey on several representative examples of Grögerregular sets.

A schematic description for Gröger-regular set is given by Fig. 2 below.

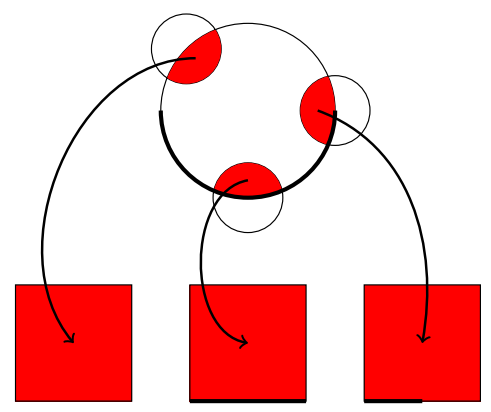

Figure 2: A schematic description of a Gröger-regular set. From left to right: the sets $M_{1}, M_{2}$ and $M_{3}$.

\subsubsection{Polyhedral domains}

We follow 32 to define a polyhedral domain. The definitions of a dihedron and a cone will also be given, which form part of the definition of a polyhedral domain.

Definiton 3.3 (Dihedron). Let $(r, \phi) \in(0, \infty) \times(0,2 \pi]$ be the polar coordinate of a non-zero two dimensional point $x^{\prime}=\left(x_{1}, x_{2}\right)$, i.e. $r=\left|x^{\prime}\right|$ and $x^{\prime}=$ $(r \cos \phi, r \sin \phi)^{T}$. The two dimensional wedge $\mathcal{W}$ with opening angle $\theta \in(0,2 \pi]$ is defined by

$$
\mathcal{W}:=\left\{x^{\prime}=\left(x_{1}, x_{2}\right): r \in(0, \infty), \phi \in(0, \theta)\right\} .
$$

The dihedron $\mathcal{D} \subset \mathbb{R}^{3}$ with opening angle $\theta$ is then defined by $\mathcal{D}:=\mathcal{W} \times \mathbb{R}$. 
Definiton 3.4 (Cone). A cone $\mathcal{K} \subset \mathbb{R}^{3}$ is defined by

$$
\mathcal{K}:=\left\{x \in \mathbb{R}^{3}-\{0\}: x /|x| \in \Gamma\right\},
$$

where $\Gamma$ is a subdomain on the unit sphere $\mathbb{S}^{2}$ such that

$$
\partial \Gamma=\bar{\Gamma}_{1} \cup \ldots \cup \bar{\Gamma}_{k}
$$

for some $k \in \mathbb{N}_{\geq 3}$, where $\Gamma_{i}$ are pairwise disjoint and non-curvewise collinear open arcs (namely, the intersection point of two different arcs has locally different tangents along the arcs) on the unit sphere.

Definiton 3.5 (Polyhedral domain). A bounded domain $\Omega \subset \mathbb{R}^{3}$ is said to be a polyhedral domain, if

- The boundary $\partial \Omega$ is a disjoint union of smooth open two dimensional manifolds $\mathcal{F}_{j}, j=1, \ldots, l$ (the faces of $\Omega$ ), smooth open curves $K_{j}, j=$ $1, \ldots, m$ (the edges of $\Omega$ ) and vertices $x^{j} \in \mathbb{R}^{3}, j=1, \ldots, k$.

- For every $x \in K_{j}$ there exist a neighborhood $U_{x} \subset \mathbb{R}^{3}$ of $x$ and a $C^{\infty}$ diffeomorphism $\iota_{x}$ such that $\iota_{x}$ maps $U_{x} \cap \Omega$ onto $\mathcal{D}_{x} \cap B_{1}$, where $\mathcal{D}_{x}$ is a dihedron and $B_{1}$ is the unit ball in $\mathbb{R}^{3}$.

- For every vertex $x^{j}$ there exist a neighborhood $U_{j} \subset \mathbb{R}^{3}$ of $x^{j}$ and a $C^{\infty}$ diffeomorphism $\iota_{j}$ such that $\iota_{j}$ maps $U_{j} \cap \Omega$ onto $\mathcal{K}_{j} \cap B_{1}$, where $\mathcal{K}_{j}$ is a cone.

\subsection{Function spaces}

The Sobolev space $W^{k, p}(\Omega)$ with non-negative integer $k$ and real number $p \in$ $[1, \infty]$ is defined by

$$
W^{k, p}(\Omega):=\left\{f \in L^{p}(\Omega): D^{\beta} f \in L^{p}(\Omega) \text { for }|\beta| \leq k\right\}
$$

with the norm

$$
\|f\|_{W^{k, p}(\Omega)}:=\left(\sum_{|\beta| \leq k}\left\|D^{\beta} f\right\|_{L^{p}(\Omega)}^{p}\right)^{\frac{1}{p}},
$$

where $D^{\beta}$ denotes the multi-differential symbol for a non-negative integer multiindex $\beta$. For a closed set $\Gamma \subset \partial \Omega$, the space $W_{\Gamma}^{1, p}(\Omega)$ with $p \in(1, \infty)$ is defined as the closure of the space

$$
\left\{\left.\phi\right|_{\Omega}: \phi \in C_{0}^{\infty}\left(\mathbb{R}^{d}\right), \operatorname{supp}(\phi) \cap \Gamma=\varnothing\right\}
$$

in the space $W^{1, p}(\Omega)$ w.r.t. the $W^{1, p}$-norm. The dual space of $W_{\Gamma}^{1, p}(\Omega)$ is denoted by $W_{\Gamma}^{-1, p^{\prime}}(\Omega)$, where $p^{\prime}$ is the Hölder conjugate of $p$ with $\frac{1}{p}+\frac{1}{p^{\prime}}=1$. We also write $H_{\Gamma}^{1}(\Omega)=W_{\Gamma}^{1,2}(\Omega)$ and its dual is denoted by $H_{\Gamma}^{-1}(\Omega)$. For $\Gamma=\partial \Omega$, $W_{\Gamma}^{1, p}(\Omega)$ and $H_{\Gamma}^{1}(\Omega)$ are denoted by $W_{0}^{1, p}(\Omega)$ and $H_{0}^{1}(\Omega)$ and their dual spaces are denoted by $W^{-1, p^{\prime}}(\Omega)$ and $H^{-1}(\Omega)$ respectively. For $f \in\left(L^{p}(\Omega)\right)^{d}$ the distributional divergence operator Div : $\left(L^{p}(\Omega)\right)^{d} \rightarrow W_{\Gamma}^{-1, p}(\Omega)$ is defined by

$$
\operatorname{Div} f[g]:=-\int_{\Omega} f \cdot \nabla g d x
$$


for all $g \in W_{\Gamma}^{1, p^{\prime}}(\Omega)$. Throughout the paper, we will also use the following shorthand notation of function spaces:

$$
\begin{aligned}
\mathbb{W}_{p} & :=\left(W_{\Gamma_{\mathbf{u}}}^{1, p}(\Omega)\right)^{d} \times W_{\Gamma_{\phi}}^{1, p}(\Omega), \\
\mathbb{W}_{p}^{*} & :=\left(W_{\Gamma_{\mathbf{u}}}^{-1, p}(\Omega)\right)^{d} \times W_{\Gamma_{\phi}}^{-1, p}(\Omega), \\
\mathbb{V}_{p, r} & :=\left(\left(W_{\Gamma_{\mathbf{P}}}^{1, p}(\Omega)\right)^{d},\left(W_{\Gamma_{\mathbf{P}}}^{-1, p}(\Omega)\right)^{d}\right)_{\frac{1}{r}, r}, \\
\mathbb{U} & :=\left\{\mathbf{P} \in(C(\bar{\Omega}))^{d}:\left.\mathbf{P}\right|_{\Gamma_{\mathbf{P}}}=0\right\} .
\end{aligned}
$$

\subsection{Real and complex interpolation}

Let $\theta, s \in(0,1), q \in[1, \infty]$ and let $X, Y$ be compatible Banach spaces in the sense of [6, Chap. 2.3]. Then $(X, Y)_{\theta, q}$ and $[X, Y]_{s}$ denote the real and complex interpolation space induced by $(X, Y)$ of components $(\theta, q)$ and of component $s$ respectively. Due to their cumbersome construction we refer to 6 , 44 for the definitions and properties of real and complex interpolation spaces.

\section{Proof of the local well-posedness result}

\subsection{An abstract existence and uniqueness result for quais- linear parabolic equations}

Our local well-posedness result relies on the existence and uniqueness result given by [7. To introduce the result, we need the following definition:

Definiton 4.1 (Maximal parabolic regularity). Let $J=\left(T_{0}, T_{1}\right)$ be a time interval. Let $X$ be a Banach space and $A: \operatorname{dom}(A) \rightarrow X$ be a closed linear operator with dense domain $\operatorname{dom}(A) \subset X$. Suppose $\tau \in(1, \infty)$. Then we say that $A$ has maximal parabolic $L^{\tau}(J ; X)$-regularity if and only if for every $f \in L^{\tau}(J ; X)$ there is a unique function $\mathbf{w} \in W^{1, \tau}(J ; X) \cap L^{\tau}(J ; \operatorname{dom}(A))$ which satisfies $\mathbf{w}\left(T_{0}\right)=0$ and

$$
\mathbf{w}^{\prime}(t)+A \mathbf{w}(t)=f(t) \quad \text { in } X
$$

for a.a. $t \in J$.

Remark 4.2. It is shown in 11 that if $A$ has maximal parabolic $L^{\tau_{0}}(J ; X)$ regularity for some $\tau_{0} \in(1, \infty)$, then it has maximal parabolic $L^{\tau}(J ; X)$-regularity for all $\tau \in(1, \infty)$. It is therefore unambiguous to merely say that $A$ has maximal parabolic regularity.

The maximal parabolic regularity property motivates the following existence and unique result introduced in [7] (see also 39]) for a quasilinear parabolic system, which plays the main role in the analysis of our local well-posedness result:

Theorem 4.3. Let $Y, X$ be Banach spaces, $Y \hookrightarrow X$ densely and let $\tau \in(1, \infty)$. Suppose that $A: J \times(Y, X)_{\frac{1}{\tau}, \tau} \rightarrow L(Y, X)$ is continuous and $A\left(0, \mathbf{w}_{0}\right)$ satisfies maximal parabolic regularity on $X$ with $\operatorname{dom}\left(A\left(0, \mathbf{w}_{0}\right)\right)=Y$ for some $\mathbf{w}_{0} \in$ $(Y, X)_{\frac{1}{\tau}, \tau}$. Let $S: J \times(Y, X)_{\frac{1}{\tau}, \tau} \rightarrow X$ be a Carathéodory map, i.e., $S(\cdot, x)$ is 
Bochner-measurable for each $x \in(Y, X)_{\frac{1}{,}, \tau}$ and $S(t, \cdot)$ is continuous for a.a. $t \in J$. Moreover, let $S(\cdot, 0)$ be from $L^{\tau}(J ; X)$ and the following assumptions be satisfied:

(A) For every $M>0$, there exists a positive constant $C_{M}$ such that for all $t \in J$ and all $\mathbf{w}, \overline{\mathbf{w}} \in(Y, X)_{\frac{1}{\tau}, \tau}$ with $\max \left(\|\mathbf{w}\|_{(Y, X)_{\frac{1}{\tau}, \tau}},\|\overline{\mathbf{w}}\|_{(Y, X)_{\frac{1}{\tau}, \tau}}\right) \leq M$, we have

$$
\|A(t, \mathbf{w})-A(t, \overline{\mathbf{w}})\|_{L(Y, X)} \leq C_{M}\|\mathbf{w}-\overline{\mathbf{w}}\|_{(Y, X)_{\frac{1}{\tau}, \tau}}
$$

(S) For every $M>0$ there exists a function $h_{M} \in L^{\tau}(J)$ such that for all $\mathbf{w}, \overline{\mathbf{w}} \in(Y, X)_{\frac{1}{\tau}, \tau}$ with $\max \left(\|\mathbf{w}\|_{(Y, X)_{\frac{1}{\tau}, \tau}},\|\overline{\mathbf{w}}\|_{(Y, X)_{\frac{1}{\tau}, \tau}}\right) \leq M$, it is true that

$$
\|S(t, \mathbf{w})-S(t, \overline{\mathbf{w}})\|_{X} \leq h_{M}(t)\|\mathbf{w}-\overline{\mathbf{w}}\|_{(Y, X)_{\frac{1}{\tau}, \tau}}
$$

for a.a. $t \in J$.

Then there exists some maximal $T_{\max } \in J$ such that the problem

$$
\mathbf{w}^{\prime}(t)+A(t, \mathbf{w}(t)) \mathbf{w}(t)=S(t, \mathbf{w}(t)) \quad \text { in } J \times X
$$

with $\mathbf{w}\left(T_{0}\right)=\mathbf{w}_{0}$ admits a unique solution

$$
\mathbf{w} \in W^{1, \tau}(0, S ; X) \cap L^{\tau}(0, S ; Y)
$$

on $(0, S)$ for every $S \in\left(0, T_{\max }\right)$. The maximality of $T_{\max }$ is understood as follows:

- Either (4.1) does not hold for $S=T_{\max }$, or

- $T_{\max }=T_{1}$ and (4.1) also holds for $S=T_{1}$.

\subsection{Embedding results for interpolation spaces}

The interpolation space $\mathbb{V}_{p, r}$ defined by (3.3) displays a somehow complicated structure at the first glance, due to the cumbersome definition of interpolation spaces. We show that $\mathbb{V}_{p, r}$ is continuously embedded into some Hölder space, which will simplify several arguments in the proof of the main results.

Lemma 4.4. Let the Assumption (A1) be satisfied. Then for all $p \in(d, \infty)$ and $r \in\left(\frac{2 p}{p-d}, \infty\right)$ we have the continuous embedding

$$
\mathbb{V}_{p, r} \hookrightarrow\left(W_{\Gamma_{\mathbf{P}}}^{1-2 \mathfrak{p}, p}(\Omega)\right)^{d}
$$

for some $\mathfrak{p} \in\left(\frac{1}{r}, \frac{p-d}{2 p}\right)$. Consequently, we have the embedding

$$
\left(W_{\Gamma_{\mathbf{P}}}^{1-2 \mathfrak{p}, p}(\Omega)\right)^{d} \hookrightarrow\left(C^{\delta}(\bar{\Omega})\right)^{d}
$$

with $\delta=1-2 \mathfrak{p}-\frac{d}{p} \in(0,1)$. 
Proof. The statement is originally proved in [34, Lem. A.1] for the case $d=3$ and $\Gamma_{\mathbf{P}}=\varnothing$. We adopt the idea therein to prove the statement for our model. From the condition $\mathfrak{p} \in\left(\frac{1}{r}, \frac{p-d}{2 p}\right)$ we obtain that

$$
\delta=1-2 \mathfrak{p}-\frac{d}{p} \in(0,1) .
$$

Thus (4.3) follows immediately from Rellich's embedding theorem. It is left to show that there exists some $\mathfrak{p} \in\left(\frac{1}{r}, \frac{p-d}{2 p}\right)$ such that

$$
\mathbb{V}_{p, r} \hookrightarrow\left(W_{\Gamma_{\mathbf{P}}}^{1-2 \mathfrak{p}, p}(\Omega)\right)^{d}
$$

Let $\nu \in\left(0, \frac{p-d}{2 p}\right)$. We obtain that

$$
\begin{aligned}
& \left(\left(W_{\Gamma_{\mathbf{P}}}^{1, p}(\Omega)\right)^{d},\left(W_{\Gamma_{\mathbf{P}}}^{-1, p}(\Omega)\right)^{d}\right)_{\nu, 1} \\
= & \left(\left(W_{\Gamma_{\mathbf{P}}}^{1, p}(\Omega)\right)^{d},\left(\left(W_{\Gamma_{\mathbf{P}}}^{1, p}(\Omega)\right)^{d},\left(W_{\Gamma_{\mathbf{P}}}^{-1, p}(\Omega)\right)^{d}\right)_{\frac{1}{2}, 1}\right)_{2 \nu, 1} \\
= & \left(\left(W_{\Gamma_{\mathbf{P}}}^{1, p}(\Omega)\right)^{d},\left(\left(W_{\Gamma_{\mathbf{P}}}^{-1, p}(\Omega)\right)^{d},\left(W_{\Gamma_{\mathbf{P}}}^{1, p}(\Omega)\right)^{d}\right)_{\frac{1}{2}, 1}\right)_{2 \nu, 1} \\
= & \left(\left(W_{\Gamma_{\mathbf{P}}}^{1, p}(\Omega)\right)^{d},\left(\left(W_{\Gamma_{\mathbf{P}}}^{-1, p}(\Omega)\right)^{d}, \operatorname{dom}(-\Delta+1)\right)_{\frac{1}{2}, 1}\right)_{2 \nu, 1},
\end{aligned}
$$

where the first equality comes from the reiteration theorem given in [44, Chap. 1.10.2], the second from the property

$$
(X, Y)_{\theta, q}=(Y, X)_{1-\theta, q}
$$

of real interpolation spaces, and the last equality from the fact that

$$
\left(W_{\Gamma_{\mathbf{P}}}^{1, p}(\Omega)\right)^{d}=\operatorname{dom}(-\Delta+1)
$$

where $\operatorname{dom}(-\Delta+1)$ is defined as the domain of the operator

$$
-\Delta+1:\left(W_{\Gamma_{\mathbf{P}}}^{1, p}(\Omega)\right)^{d} \rightarrow\left(W_{\Gamma_{\mathbf{P}}}^{-1, p}(\Omega)\right)^{d} .
$$

According to [4, Thm. 11.5], the extension of the operator $-\Delta+1$ from $\left(L^{p}(\Omega)\right)^{d}$ to $\left(W_{\Gamma_{\mathbf{P}}}^{-1, p}(\Omega)\right)^{d}$ is a positive operator in the sense of [44, Chap. 1.14]. This property enables us to exploit [44, Chap. 1.15.2] to define the square root $(-\Delta+1)^{\frac{1}{2}}$ of $-\Delta+1$ on $\left(W_{\Gamma_{\mathbf{P}}}^{-1, p}(\Omega)\right)^{d}$ with domain $\operatorname{dom}\left((-\Delta+1)^{\frac{1}{2}}\right)$ and to obtain that

$$
\left(\left(W_{\Gamma_{\mathbf{P}}}^{-1, p}(\Omega)\right)^{d}, \operatorname{dom}(-\Delta+1)\right)_{\frac{1}{2}, 1} \hookrightarrow \operatorname{dom}\left((-\Delta+1)^{\frac{1}{2}}\right) .
$$

From [4, Thm. 5.1 (ii)] one infers the embedding

$$
\operatorname{dom}\left((-\Delta+1)^{\frac{1}{2}}\right) \hookrightarrow\left(L^{p}(\Omega)\right)^{d}
$$

Finally, from (4.6) and (4.7) we conclude that

$$
\left(\left(W_{\Gamma_{\mathbf{P}}}^{-1, p}(\Omega)\right)^{d}, \operatorname{dom}(-\Delta+1)\right)_{\frac{1}{2}, 1} \hookrightarrow\left(L^{p}(\Omega)\right)^{d} .
$$


It follows that

$$
\begin{aligned}
& \left(\left(W_{\Gamma_{\mathbf{P}}}^{1, p}(\Omega)\right)^{d},\left(W_{\Gamma_{\mathbf{P}}}^{-1, p}(\Omega)\right)^{d}\right)_{\nu, 1} \\
\hookrightarrow & \left(\left(W_{\Gamma_{\mathbf{P}}}^{1, p}(\Omega)\right)^{d},\left(L^{p}(\Omega)\right)^{d}\right)_{2 \nu, 1} \\
= & \left(\left(L^{p}(\Omega)\right)^{d},\left(W_{\Gamma_{\mathbf{P}}}^{1, p}(\Omega)\right)^{d}\right)_{1-2 \nu, 1} \\
\hookrightarrow & {\left[\left(L^{p}(\Omega)\right)^{d},\left(W_{\Gamma_{\mathbf{P}}}^{1, p}(\Omega)\right)^{d}\right]_{1-2 \nu}=\left(W_{\Gamma_{\mathbf{P}}}^{1-2 \nu, p}(\Omega)\right)^{d}, }
\end{aligned}
$$

where the first embedding comes from (4.4) and (4.8), the first equality is obtained by using (4.5), the second embedding comes from the property

$$
(X, Y)_{\theta, 1} \hookrightarrow[X, Y]_{\theta}
$$

of interpolation spaces, and the second equality is deduced from [14, Thm. 3.1] (notice that $\nu$ is in $\left(0, \frac{p-d}{2 p}\right)$, which particularly implies that $1-2 \nu>\frac{d}{p}>\frac{1}{p}$ and consequently that $1-2 \nu \neq \frac{1}{p}$, being the condition of [14, Thm. 3.1]). Finally, using the fundamental properties of real interpolation spaces we obtain that

$$
\begin{aligned}
& \left(\left(W_{\Gamma_{\mathbf{P}}}^{1, p}(\Omega)\right)^{d},\left(W_{\Gamma_{\mathbf{P}}}^{-1, p}(\Omega)\right)^{d}\right)_{\frac{1}{r}, r} \\
\hookrightarrow & \left(\left(W_{\Gamma_{\mathbf{P}}}^{1, p}(\Omega)\right)^{d},\left(W_{\Gamma_{\mathbf{P}}}^{-1, p}(\Omega)\right)^{d}\right)_{\nu, 1} \\
\hookrightarrow & \left(W_{\Gamma_{\mathbf{P}}}^{1-2 \nu, p}(\Omega)\right)^{d}
\end{aligned}
$$

for $\nu \in\left(\frac{1}{r}, 1\right)$, where the first embedding follows from [44, 1.3.3. (4)]. The condition on $r$ implies that the interval $\left(\frac{1}{r}, \frac{p-d}{2 p}\right)$ is not empty, thus one can choose some $\mathfrak{p} \in\left(\frac{1}{r}, \frac{p-d}{2 p}\right) \subset\left(0, \frac{p-d}{2 p}\right)$ such that (4.9) is valid. This completes the proof.

The following proposition reveals the temporal and spatial Hölder continuity of a local solution $\mathbf{P}$.

Proposition 4.5. Let the Assumption (A1) be satisfied. Then for $p \in(d, \infty)$ and $r \in\left(\frac{2 p}{p-d}, \infty\right)$ there exists some $\delta \in(0,1)$ such that the embedding

$$
W^{1, r}\left(J ;\left(W_{\Gamma_{\mathbf{P}}}^{-1, p}(\Omega)\right)^{d}\right) \cap L^{r}\left(J ;\left(W_{\Gamma_{\mathbf{P}}}^{1, p}(\Omega)\right)^{d}\right) \hookrightarrow C^{\delta}\left(\bar{J} ;\left(C^{\delta}(\bar{\Omega})\right)^{d}\right)
$$

is valid for each interval $J \subset \mathbb{R}$.

Proof. From [3, Thm. 3] we have the embedding

$$
\begin{aligned}
& W^{1, r}\left(J ;\left(W_{\Gamma_{\mathbf{P}}}^{-1, p}(\Omega)\right)^{d}\right) \cap L^{r}\left(J ;\left(W_{\Gamma_{\mathbf{P}}}^{1, p}(\Omega)\right)^{d}\right) \\
\hookrightarrow & C^{s-\frac{1}{r}}\left(\bar{J} ;\left(\left(W_{\Gamma_{\mathbf{P}}}^{-1, p}(\Omega), W_{\Gamma_{\mathbf{P}}}^{1, p}(\Omega)\right)_{\theta, 1}\right)^{d}\right)
\end{aligned}
$$

for $s \in\left(\frac{1}{r}, 1\right)$ and $\theta \in[0,1-s)$. Notice that $1-\theta \in(s, 1]$ and $s \in\left(\frac{1}{r}, 1\right)$ and $1-\theta, s$ can be arbitrarily chosen in these intervals. The condition on $r$ implies that $\frac{1}{r}<\frac{p-d}{2 p}$, thus one can choose $s$ sufficiently small and then $\theta$ sufficiently large such that $s \in\left(\frac{1}{r}, \frac{p-d}{2 p}\right)$ and $1-\theta \in\left(s, \frac{p-d}{2 p}\right] \subset\left(0, \frac{p-d}{2 p}\right)$. Then (4.9) is satisfied and we obtain that

$$
\left(W_{\Gamma_{\mathbf{P}}}^{-1, p}(\Omega), W_{\Gamma_{\mathbf{P}}}^{1, p}(\Omega)\right)_{\theta, 1}=\left(W_{\Gamma_{\mathbf{P}}}^{1, p}(\Omega), W_{\Gamma_{\mathbf{P}}}^{-1, p}(\Omega)\right)_{1-\theta, 1}
$$




$$
\hookrightarrow C^{1-2(1-\theta)-\frac{d}{p}}(\bar{\Omega}) .
$$

By choosing $\delta:=\min \left\{s-\frac{1}{r}, 1-2(1-\theta)-\frac{d}{p}\right\} \in(0,1)$ we obtain the desired result.

\subsection{Uniform boundedness of $\mathrm{L}_{\mathbf{P}}^{-1}$}

Let the number $p$ and the space $\mathbb{U}$ be as defined by Assumption (A3) and let $(\mathbf{u}, \phi)$ be the solution of the differential equation

$$
\mathrm{L}_{\mathbf{P}}(\mathbf{u}, \phi)=\ell
$$

for $\mathbf{P} \in \mathbb{U}$ and $\ell \in \mathbb{W}_{p}^{*}$. Then from Assumption (A3) we have the estimate

$$
\|(\mathbf{u}, \phi)\|_{\mathbb{W}_{p}} \leq C_{\mathbf{P}}\|\ell\|_{\mathbb{W}_{p}^{*}},
$$

where $C_{\mathbf{P}}=\left\|\mathrm{L}_{\mathbf{P}}^{-1}\right\|_{L\left(\mathbb{W}_{p}^{*}, \mathbb{W}_{p}\right)}$ is the inverse operator norm. Under the Assumptions (B1) to (B3) the constant $C_{\mathbf{P}}$ is uniform for all test functions $\mathbf{P} \in \mathbb{U}$, which is a direct consequence of Theorem 5.2, In general, however, the inverse operator norm $C_{\mathbf{P}}$ also depends on the modulus of continuity of $\mathbb{B}(\mathbf{P})$, thus $C_{\mathbf{P}}$ could tend to infinity by testing various $\mathbf{P}$ even when they are uniformly bounded in $\mathbb{U}$, which makes the verification of the condition (S) of Theorem 4.3 impossible. From Lemma 4.4 we know yet that all test functions $\mathbf{P}$ will be taken from a bounded subset of a Hölder space. Using the compact embedding of a Hölder space into $(C(\bar{\Omega}))^{d}$ and the indirect continuity arguments given by [34] we are able to show the uniform boundedness of $C_{\mathbf{P}}$.

Lemma 4.6. Let the Assumptions (A1) to (A3) be satisfied and let $p$ be the number given by Assumption (A3), Let $\mathcal{M}$ be a compact subset of the space $\mathbb{U}$. Then the norm $C_{\mathbf{P}}$ of the inverse operator $\mathrm{L}_{\mathbf{P}}^{-1}$ is uniformly bounded by some positive constant $C^{*}$ for all $\mathbf{P} \in \mathcal{M}$.

Proof. Firstly we show that the mapping

$$
\mathbb{U} \ni \mathbf{P} \mapsto J(\mathbf{P}):=\left(-\operatorname{Div}\left(\mathbb{B}(\mathbf{P})\left(\begin{array}{c}
\varepsilon\left(\left.\cdot\right|_{\mathbf{u}}\right) \\
\left.\nabla \cdot\right|_{\phi}
\end{array}\right)\right)\right)^{-1} \in L H\left(\mathbb{W}_{p}^{*}, \mathbb{W}_{p}\right)
$$

is well-defined and continuous, where $L H(X, Y)$ denotes the set of linear homeomorphisms between Banach spaces $X$ and $Y$. On the one hand, we obtain that

$$
\begin{aligned}
& \mathrm{L}_{\mathbf{P}_{1}}(\mathbf{u}, \phi)[(\overline{\mathbf{u}}, \bar{\phi})]-\mathrm{L}_{\mathbf{P}_{2}}(\mathbf{u}, \phi)[(\overline{\mathbf{u}}, \bar{\phi})] \\
= & \int_{\Omega}\left(\mathbb{B}\left(\mathbf{P}_{1}\right)-\mathbb{B}\left(\mathbf{P}_{2}\right)\right)\left(\begin{array}{c}
\varepsilon(\mathbf{u}) \\
\nabla \phi
\end{array}\right):\left(\begin{array}{c}
\varepsilon(\overline{\mathbf{u}}) \\
\nabla \bar{\phi}
\end{array}\right) d x \\
\leq & C_{1}^{*} \int_{\Omega}\left|\mathbf{P}_{1}-\mathbf{P}_{2}\right| \cdot|(\varepsilon(\mathbf{u}), \nabla \phi)| \cdot|(\varepsilon(\overline{\mathbf{u}}), \nabla \bar{\phi})| d x \\
\leq & C C_{1}^{*}\left\|\mathbf{P}_{1}-\mathbf{P}_{2}\right\|_{L^{\infty}(\Omega)}\|(\mathbf{u}, \phi)\|_{\mathbb{W}_{p}}\|(\overline{\mathbf{u}}, \bar{\phi})\|_{\mathbb{W}_{p^{\prime}}}
\end{aligned}
$$

for all $(\mathbf{u}, \phi) \in \mathbb{W}_{p}$ and $(\overline{\mathbf{u}}, \bar{\phi}) \in \mathbb{W}_{p^{\prime}}$, where $C_{1}^{*}$ denotes the Lipschitz constant of $\mathbb{B}$ on the closed ball $\bar{B}_{M}(0) \subset \mathbb{R}^{d}$, and $M$ is the upper bound of $\mathcal{M}$ in $\mathbb{U}$. 
From this we infer that the mapping

$$
\mathbb{U} \ni \mathbf{P} \mapsto-\operatorname{Div}\left(\mathbb{B}(\mathbf{P})\left(\begin{array}{c}
\varepsilon\left(\left.\cdot\right|_{\mathbf{u}}\right) \\
\left.\nabla \cdot\right|_{\phi}
\end{array}\right)\right) \in L H\left(\mathbb{W}_{p}, \mathbb{W}_{p}^{*}\right)
$$

is continuous (that the image is in fact a linear homeomorphism follows from Assumption (A3)]. On the other hand, from fundamental analysis we know that for Banach spaces $X$ and $Y$, the mapping $L H(X, Y) \ni B \mapsto B^{-1} \in$ $L H(Y, X)$ is continuous. Then the continuity of (4.11) follows, as the mapping $J$ is a composition of continuous functions. Now since $J$ is continuous and $\mathcal{M}$ is compact, we see that $J(\mathcal{M})$ is a compact subset of $L\left(\mathbb{W}_{p}^{*}, \mathbb{W}_{p}\right)$. In particular we obtain that

$$
C^{*}=\sup _{\mathbf{P} \in \mathcal{M}}\|J(\mathbf{P})\|_{L\left(\mathbb{W}_{p}^{*}, \mathbb{W}_{p}\right)}<\infty .
$$

This completes the proof.

\subsection{Proof of Theorem 2.3}

We are now ready to prove Theorem 2.3 ,

Proof of Theorem 2.3. Let us first recall the following function spaces

$$
\begin{aligned}
\mathbb{W}_{p} & =\left(W_{\Gamma_{\mathbf{u}}}^{1, p}(\Omega)\right)^{d} \times W_{\Gamma_{\phi}}^{1, p}(\Omega), \\
\mathbb{W}_{p}^{*} & =\left(W_{\Gamma_{\mathbf{u}}}^{-1, p}(\Omega)\right)^{d} \times W_{\Gamma_{\phi}}^{-1, p}(\Omega), \\
\mathbb{V}_{p, r} & =\left(\left(W_{\Gamma_{\mathbf{P}}}^{1, p}(\Omega)\right)^{d},\left(W_{\Gamma_{\mathbf{P}}}^{-1, p}(\Omega)\right)^{d}\right)_{\frac{1}{r}, r}, \\
\mathbb{U} & =\left\{\overline{\mathbf{P}} \in(C(\bar{\Omega}))^{d}: \overline{\mathbf{P}}_{\Gamma_{\mathbf{P}}}=0\right\}
\end{aligned}
$$

defined by (3.1) to (3.4), which will be extensively used in the remaining proof. We formulate the following notation corresponding to the ones given by Theorem 4.3

$$
\begin{aligned}
A(t, \mathbf{P}) & \equiv-\Delta, \\
\tau & =r \\
Y & =\left(W_{\Gamma_{\mathbf{P}}}^{1, p}(\Omega)\right)^{d}, \\
X & =\left(W_{\Gamma_{\mathbf{P}}}^{-1, p}(\Omega)\right)^{d}
\end{aligned}
$$

and

$$
S(t, \mathbf{P})=F\left(t, \varepsilon\left(\mathbf{u}_{\mathbf{P}}(t)\right), \nabla \phi_{\mathbf{P}}(t), \mathbf{P}\right)
$$

where $\left(\mathbf{u}_{\mathbf{P}}(t), \phi_{\mathbf{P}}(t)\right)$ is the unique $\mathbb{W}_{p^{-}}$-weak solution of the differential equation

$$
\mathrm{L}_{\mathbf{P}}\left(\mathbf{u}_{\mathbf{P}}(t), \phi_{\mathbf{P}}(t)\right)=\ell(t, \mathbf{P})
$$

for $\mathbf{P} \in(Y, X)_{\frac{1}{\tau}, \tau}=\mathbb{V}_{p, r} \subset \mathbb{U}$ (the inclusion is deduced from Lemma 4.4), with $\ell(t, \mathbf{P})$ and $F(t, \varepsilon, \nabla \phi, \mathbf{P})$ given by (2.6), (2.7) respectively. In particular, $\left(\mathbf{u}_{\mathbf{P}}(t), \phi_{\mathbf{P}}(t)\right)$ is uniquely determined by a given pair $(t, \mathbf{P})$, thus $S(t, \mathbf{P})$ is welldefined. Having defined these notation, we utilize Theorem 4.3 to show that the equation

$$
\mathbf{P}^{\prime}(t)+A(t, \mathbf{P}(t))=S(t, \mathbf{P}(t))
$$


with initial value $\mathbf{P}_{0}$ has a unique local solution $\mathbf{P}$. We firstly give the following statements corresponding to part of the conditions from Theorem 4.3, which are relatively easier to verify:

- That $Y \hookrightarrow X$ densely is trivial.

- That $A\left(0, \mathbf{P}_{0}\right)=-\Delta$ satisfies maximal parabolic regularity on $X$ with $\operatorname{dom}\left(A\left(0, \mathbf{P}_{0}\right)\right)=Y$ follows from [19, Thm. 5.4, Rem. 5.5], where by applying [12, Thm. 1.2] the volume-preserving condition in [19] can be weakened to the $(d-1)$-Ahlfors-David-regular condition.

- That $A(t, \mathbf{P})$ is continuous from $[0, T] \times(Y, X)_{\frac{1}{\tau}, \tau}$ to $L(Y, X)$ is trivial, since $A$ is constantly equal to $-\Delta$.

- Since $A$ is constantly equal to $-\Delta$, the validity of (A) in Theorem 4.3 is evident.

- Now we show that

$$
S(t, \cdot):(Y, X)_{\frac{1}{\tau}, \tau} \rightarrow X
$$

is continuous for a.a. $t \in[0, T]$. Let $\mathbf{P} \in(Y, X)_{\frac{1}{\tau}, \tau}$ be arbitrary. For $\kappa>0$ define

$$
\mathcal{O}_{\kappa}(\mathbf{P}):=\left\{\overline{\mathbf{P}} \in(Y, X)_{\frac{1}{\tau}, \tau}:\|\mathbf{P}-\overline{\mathbf{P}}\|_{(Y, X)_{\frac{1}{\tau}, \tau}}<\kappa\right\} .
$$

Thus to show the continuity, it suffices to show that

$$
\lim _{\kappa \rightarrow 0} \sup _{\overline{\mathbf{P}} \in \mathcal{O}_{\kappa}(\mathbf{P})}\|S(t, \mathbf{P})-S(t, \overline{\mathbf{P}})\|_{X}=0 .
$$

But this follows directly from the Assumption (S) given in Theorem 4.3, which will be shown in the subsequent part of the proof below and we do not repeat here.

It is left to show that

- given a fixed $\mathbf{P}$ in $(Y, X)_{\frac{1}{\tau}, \tau}$, the mapping $t \mapsto S(t, \mathbf{P})$ is Bochner-measurable,

- the validity of (S) in Theorem 4.3 and

- $S(\cdot, 0)$ is from $L^{r}(0, T ; X)$.

We will show these statements in the following steps.

Step 1: Bochner-measurability of $t \mapsto S(t, \mathbf{P})$

Recall that

$$
\begin{aligned}
S(t, \mathbf{P}) & =F\left(t, \varepsilon\left(\mathbf{u}_{\mathbf{P}}(t)\right), \nabla \phi_{\mathbf{P}}(t), \mathbf{P}\right) \\
& =-D_{\mathbf{P}} H\left(\varepsilon\left(\mathbf{u}_{\mathbf{P}}(t)\right), \nabla \phi_{\mathbf{P}}(t), \mathbf{P}\right)-D_{\mathbf{P}} \omega(\mathbf{P})+\ell_{3}(t) .
\end{aligned}
$$

The Bochner-measurability of $t \mapsto \ell_{3}(t)$ follows directly from Assumption (A4), and $t \mapsto D_{\mathbf{P}} \omega(\mathbf{P})$ is also Bochner-measurable since it is time-independent. We thus still need to verify the Bochner-measurability of the mapping $t \mapsto$ 
$D_{\mathbf{P}} H\left(\varepsilon\left(\mathbf{u}_{\mathbf{P}}(t)\right), \nabla \phi_{\mathbf{P}}(t), \mathbf{P}\right)$. We firstly show the Bochner-measurability of the mapping $t \mapsto\left(\mathbf{u}_{\mathbf{P}}(t), \phi_{\mathbf{P}}(t)\right)$. This means that we need to find a sequence $\left(\mathbf{u}_{n}(t), \phi_{n}(t)\right)$ of simple functions such that

$$
\left(\mathbf{u}_{n}(t), \phi_{n}(t)\right) \rightarrow\left(\mathbf{u}_{\mathbf{P}}(t), \phi_{\mathbf{P}}(t)\right) \quad \text { in } \mathbb{W}_{p} \text { for a.a. } t \in[0, T]
$$

as $n \rightarrow \infty$. Recall from (2.6) that $\ell(t, \mathbf{P})$ is equal to

$$
\ell(t, \mathbf{P})=\ell_{1}(\mathbf{P})+\ell_{2}(t)
$$

where $\ell_{1}$ and $\ell_{2}$ are given by (2.8) and (2.9) respectively. Since $\ell_{2}$ is Bochnermeasurable, there exist simple functions $\left\{\ell_{2}^{n}\right\}_{n \in \mathbb{N}}$ and some measurable set $\Theta \subset$ $[0, T]$ with full measure such that

$$
\ell_{2}^{n}(t) \rightarrow \ell_{2}(t) \quad \text { in } \mathbb{W}_{p}^{*} \text { for all } t \in \Theta
$$

as $n \rightarrow \infty$. Now let $t \in \Theta$. It is immediate that $\ell_{1}(\mathbf{P})$ is an element of $\mathbb{W}_{p}^{*}$ for each $\mathbf{P} \in(Y, X)_{\frac{1}{\tau}, \tau} \subset(C(\bar{\Omega}))^{d}$ by using Assumption $(\mathrm{A} 2)$. Hence Assumption (A3) is applicable with the r.h.s. function

$$
\ell^{n}(t, \mathbf{P}):=\ell_{1}(\mathbf{P})+\ell_{2}^{n}(t)
$$

and we denote by $\left(\mathbf{u}_{n}(t), \phi_{n}(t)\right)$ the unique $\mathbb{W}_{p}$-solution of

$$
\mathrm{L}_{\mathbf{P}}\left(\mathbf{u}_{n}(t), \phi_{n}(t)\right)=\ell^{n}(t, \mathbf{P}) .
$$

Since $\ell^{n}(t, \mathbf{P})$ is a simple function, either is $\left(\mathbf{u}_{n}(t), \phi_{n}(t)\right)$. Moreover, we obtain that

$$
\mathrm{L}_{\mathbf{P}}\left(\mathbf{u}_{n}(t)-\mathbf{u}_{\mathbf{P}}(t), \phi_{n}(t)-\phi_{\mathbf{P}}(t)\right)=\ell^{n}(t, \mathbf{P})-\ell(t, \mathbf{P})=\ell_{2}^{n}(t)-\ell_{2}(t) .
$$

This, together with (4.17) and (4.10) immediately implies 4.15). Next, we show that

$$
D_{\mathbf{P}} H\left(\varepsilon\left(\mathbf{u}_{n}(t)\right), \nabla \phi_{n}(t), \mathbf{P}\right) \rightarrow D_{\mathbf{P}} H\left(\varepsilon\left(\mathbf{u}_{\mathbf{P}}(t)\right), \nabla \phi_{\mathbf{P}}(t), \mathbf{P}\right) \quad \text { in } X \text { for all } t \in \Theta
$$

as $n \rightarrow \infty$. Since $\left\{D_{\mathbf{P}} H\left(\varepsilon\left(\mathbf{u}_{n}(t)\right), \nabla \phi_{n}(t), \mathbf{P}\right)\right\}_{n \in \mathbb{N}}$ is a sequence of simple functions, (4.19) will complete the proof of Step 1. Let $\overline{\mathbf{P}} \in\left(W_{\Gamma_{\mathbf{P}}}^{1, p^{\prime}}(\Omega)\right)^{d}$. We obtain from (1.6) that

$$
\begin{aligned}
& \int_{\Omega} D_{\mathbf{P}} H(\varepsilon(\mathbf{u}), \nabla \phi, \mathbf{P}) \cdot \overline{\mathbf{P}} d x \\
= & \int_{\Omega} \frac{1}{2} D_{\mathbf{P}} \mathbb{B}(\mathbf{P}) \overline{\mathbf{P}}\left(\begin{array}{c}
\varepsilon(\mathbf{u})-\varepsilon^{0}(\mathbf{P}) \\
-\nabla \phi
\end{array}\right):\left(\begin{array}{c}
\varepsilon(\mathbf{u})-\varepsilon^{0}(\mathbf{P}) \\
\nabla \phi
\end{array}\right) d x \\
+ & \int_{\Omega} \mathbb{B}(\mathbf{P})\left(\begin{array}{c}
-D_{\mathbf{P}} \varepsilon^{0}(\mathbf{P}) \overline{\mathbf{P}} \\
0
\end{array}\right):\left(\begin{array}{c}
\varepsilon(\mathbf{u})-\varepsilon^{0}(\mathbf{P}) \\
\nabla \phi
\end{array}\right) d x+\int_{\Omega} \nabla \phi \cdot \overline{\mathbf{P}} d x \\
= & : \mathcal{S}_{1}(\mathbf{u}, \phi, \mathbf{P})+\mathcal{S}_{2}(\mathbf{u}, \phi, \mathbf{P})+\mathcal{S}_{3}(\phi) .
\end{aligned}
$$

We firstly show that the integral in (4.20) is well-defined for $(\mathbf{u}, \phi) \in \mathbb{W}_{p}$. Using Assumption (A2) and the fact that $\mathbf{P} \in(C(\bar{\Omega}))^{d}$ we can find some $K_{\mathbf{P}} \in(1, \infty)$ such that

$$
\sup _{x \in \bar{\Omega}}\left\{|\mathbb{B}(\mathbf{P}(x))|,\left|D_{\mathbf{P}} \mathbb{B}(\mathbf{P}(x))\right|,\left|\varepsilon^{0}(\mathbf{P}(x))\right|,\left|D_{\mathbf{P}} \varepsilon^{0}(\mathbf{P}(x))\right|\right\}+\|\mathbf{P}\|_{L^{\infty}(\Omega)} \leq K_{\mathbf{P}} .
$$


We obtain that

$$
\begin{aligned}
& \left|\mathcal{S}_{1}(\mathbf{u}, \phi, \mathbf{P})+\mathcal{S}_{2}(\mathbf{u}, \phi, \mathbf{P})\right| \\
\leq & \int_{\Omega} C K_{\mathbf{P}}\left(|\varepsilon(\mathbf{u})|^{2}+|\nabla \phi|^{2}+K_{\mathbf{P}}^{2}\right)|\overline{\mathbf{P}}|+K_{\mathbf{P}}^{2}\left(|\varepsilon(\mathbf{u})|+|\nabla \phi|+K_{\mathbf{P}}\right)|\overline{\mathbf{P}}| d x \\
\leq & \int_{\Omega} C K_{\mathbf{P}}^{3}\left(1+|\varepsilon(\mathbf{u})|^{2}+|\nabla \phi|^{2}\right)|\overline{\mathbf{P}}| d x \\
\leq & C K_{\mathbf{P}}^{3}\left(\|1\|_{L^{p}(\Omega)}^{2}+\|\varepsilon(\mathbf{u})\|_{L^{p}(\Omega)}^{2}+\|\nabla \phi\|_{L^{p}(\Omega)}^{2}\right)\|\overline{\mathbf{P}}\|_{L^{\frac{p}{p-2}}(\Omega)} \\
\leq & C K_{\mathbf{P}}^{3}\left(1+\|\mathbf{u}\|_{W^{1, p}(\Omega)}^{2}+\|\phi\|_{W^{1, p}(\Omega)}^{2}\right)\|\overline{\mathbf{P}}\|_{W^{1, p^{\prime}}(\Omega)},
\end{aligned}
$$

where we used the equality $\frac{1}{p}+\frac{1}{p}+\frac{p-2}{p}=1$ for the Hölder's inequality and the fact that $W^{1, p^{\prime}}(\Omega) \hookrightarrow L^{\frac{p}{p-2}}(\Omega)$ for $p \in(d, \infty)$, which follows from the implication

$$
p \in(d, \infty) \Rightarrow 1-\frac{d}{p^{\prime}} \geq 0-d /\left(\frac{p}{p-2}\right) .
$$

Analogously, for $\mathcal{S}_{3}(\phi)$ we have

$$
\left|\mathcal{S}_{3}(\phi)\right| \leq C\|\nabla \phi\|_{L^{p}(\Omega)}\|\overline{\mathbf{P}}\|_{L^{\frac{p}{p-2}(\Omega)}} \leq C\|\phi\|_{W^{1, p}(\Omega)}\|\overline{\mathbf{P}}\|_{W^{1, p^{\prime}}(\Omega)} .
$$

The well-definedness of the integral in (4.20) then follows from the fact that $(\mathbf{u}, \phi) \in \mathbb{W}_{p} \subset\left(W^{1, p}(\Omega)\right)^{d}$. Now we consider the difference

$$
\begin{aligned}
& \int_{\Omega}\left(D_{\mathbf{P}} H\left(\varepsilon\left(\mathbf{u}_{n}(t)\right), \nabla \phi_{n}(t), \mathbf{P}\right)-D_{\mathbf{P}} H\left(\varepsilon\left(\mathbf{u}_{\mathbf{P}}(t)\right), \nabla \phi_{\mathbf{P}}(t), \mathbf{P}\right)\right) \cdot \overline{\mathbf{P}} d x \\
= & \left(\mathcal{S}_{1}\left(\mathbf{u}_{n}(t), \phi_{n}(t), \mathbf{P}\right)-\mathcal{S}_{1}\left(\mathbf{u}_{\mathbf{P}}(t), \phi_{\mathbf{P}}(t), \mathbf{P}\right)\right) \\
+ & \left(\mathcal{S}_{2}\left(\mathbf{u}_{n}(t), \phi_{n}(t), \mathbf{P}\right)-\mathcal{S}_{2}\left(\mathbf{u}_{\mathbf{P}}(t), \phi_{\mathbf{P}}(t), \mathbf{P}\right)\right) \\
+ & \left(\mathcal{S}_{3}\left(\phi_{n}(t)\right)-\mathcal{S}_{3}\left(\phi_{\mathbf{P}}(t)\right)\right) .
\end{aligned}
$$

We recall that

$$
\begin{gathered}
\mathrm{L}_{\mathbf{P}}\left(\mathbf{u}_{n}(t), \phi_{n}(t)\right)=\ell_{1}(\mathbf{P})+\ell_{2}^{n}(t), \\
\mathrm{L}_{\mathbf{P}}\left(\mathbf{u}_{\mathbf{P}}(t), \phi_{\mathbf{P}}(t)\right)=\ell_{1}(\mathbf{P})+\ell_{2}(t), \\
\mathrm{L}_{\mathbf{P}}\left(\mathbf{u}_{n}(t)-\mathbf{u}_{\mathbf{P}}(t), \phi_{n}(t)-\phi_{\mathbf{P}}(t)\right)=\ell_{2}^{n}(t)-\ell_{2}(t) .
\end{gathered}
$$

From (2.8) it follows

$$
\left\|l_{1}(\mathbf{P})\right\|_{\mathbb{W}_{p}^{*}} \leq C K_{\mathbf{P}}^{2}
$$

Applying (4.10) on (4.25) to (4.27), we deduce that

$$
\begin{aligned}
\left\|\left(\mathbf{u}_{\mathbf{P}}(t), \phi_{\mathbf{P}}(t)\right)\right\|_{\mathbb{W}_{p}} & \leq C C_{\mathbf{P}} K_{\mathbf{P}}^{2}\left(1+\left\|l_{2}(t)\right\|_{\mathbb{W}_{p}^{*}}\right), \\
\left\|\left(\mathbf{u}_{n}(t), \phi_{n}(t)\right)\right\|_{\mathbb{W}_{p}} & \leq C C_{\mathbf{P}} K_{\mathbf{P}}^{2}\left(1+\left\|l_{2}^{n}(t)\right\|_{\mathbb{W}_{p}^{*}}\right), \\
\left\|\left(\mathbf{u}_{n}(t)-\mathbf{u}_{\mathbf{P}}(t), \phi_{n}(t)-\phi_{\mathbf{P}}(t)\right)\right\|_{\mathbb{W}_{p}} & \leq C C_{\mathbf{P}}\left\|l_{2}^{n}(t)-l_{2}(t)\right\|_{\mathbb{W}_{p}^{*}},
\end{aligned}
$$


where $C_{\mathbf{P}}$ is the constant given by (4.10). Using telescoping technique and (4.29) to (4.31) we obtain that

$$
\begin{aligned}
& \left|\mathcal{S}_{1}\left(\mathbf{u}_{n}(t), \phi_{n}(t), \mathbf{P}\right)-\mathcal{S}_{1}\left(\mathbf{u}_{\mathbf{P}}(t), \phi_{\mathbf{P}}(t), \mathbf{P}\right)\right| \\
\leq & \int_{\Omega} C K_{\mathbf{P}}\left(\left|\varepsilon\left(\mathbf{u}_{n}(t)\right)-\varepsilon\left(\mathbf{u}_{\mathbf{P}}(t)\right)\right|+\left|\nabla \phi_{n}(t)-\nabla \phi_{\mathbf{P}}(t)\right|\right)|\overline{\mathbf{P}}| \\
\cdot & \left(\left|\varepsilon\left(\mathbf{u}_{n}(t)\right)\right|+\left|\varepsilon\left(\mathbf{u}_{\mathbf{P}}(t)\right)\right|+\left|\nabla \phi_{n}(t)\right|+\left|\nabla \phi_{\mathbf{P}}(t)\right|+K_{\mathbf{P}}\right) d x \\
\leq & \int_{\Omega} C K_{\mathbf{P}}^{2}\left(\left|\varepsilon\left(\mathbf{u}_{n}(t)\right)-\varepsilon\left(\mathbf{u}_{\mathbf{P}}(t)\right)\right|+\left|\nabla \phi_{n}(t)-\nabla \phi_{\mathbf{P}}(t)\right|\right)|\overline{\mathbf{P}}| \\
& \left(1+\left|\varepsilon\left(\mathbf{u}_{n}(t)\right)\right|+\left|\varepsilon\left(\mathbf{u}_{\mathbf{P}}(t)\right)\right|+\left|\nabla \phi_{n}(t)\right|+\left|\nabla \phi_{\mathbf{P}}(t)\right|\right) d x \\
\leq & C K_{\mathbf{P}}^{2}\left(\left\|\varepsilon\left(\mathbf{u}_{n}(t)\right)-\varepsilon\left(\mathbf{u}_{\mathbf{P}}(t)\right)\right\|_{L^{p}(\Omega)}+\left\|\nabla \phi_{n}(t)-\nabla \phi_{\mathbf{P}}(t)\right\|_{L^{p}(\Omega)}\right)\|\overline{\mathbf{P}}\|_{L^{\frac{p}{p-2}}(\Omega)} \\
\cdot & \left(1+\left\|\varepsilon\left(\mathbf{u}_{n}(t)\right)\right\|_{L^{p}(\Omega)}+\left\|\varepsilon\left(\mathbf{u}_{\mathbf{P}}(t)\right)\right\|_{L^{p}(\Omega)}+\left\|\nabla \phi_{n}(t)\right\|_{L^{p}(\Omega)}+\left\|\nabla \phi_{\mathbf{P}}(t)\right\|_{L^{p}(\Omega)}\right) \\
\leq & C K_{\mathbf{P}}^{4} C_{\mathbf{P}}^{2}\left\|l_{2}^{n}(t)-l_{2}(t)\right\|_{\mathbb{W}_{p}^{*}}\left(1+\left\|l_{2}^{n}(t)\right\|_{\mathbb{W}_{p}^{*}}+\left\|l_{2}(t)\right\|_{\mathbb{W}_{p}^{*}}\right)\|\overline{\mathbf{P}}\|_{W^{1, p^{\prime}}(\Omega)}, \quad(4.32) \\
& \left|\mathcal{S}_{2}\left(\mathbf{u}_{n}(t), \phi_{n}(t), \mathbf{P}\right)-\mathcal{S}_{2}\left(\mathbf{u}_{\mathbf{P}}(t), \phi_{\mathbf{P}}(t), \mathbf{P}\right)\right| \\
\leq & \int_{\Omega} K_{\mathbf{P}}^{2}\left(\left|\varepsilon\left(\mathbf{u}_{n}(t)\right)-\varepsilon\left(\mathbf{u}_{\mathbf{P}}(t)\right)\right|+\left|\phi_{n}(t)-\phi_{\mathbf{P}}(t)\right|\right)|\overline{\mathbf{P}}| d x \\
\leq & C K_{\mathbf{P}}^{2}\left(\left\|\varepsilon\left(\mathbf{u}_{n}(t)\right)-\varepsilon\left(\mathbf{u}_{\mathbf{P}}(t)\right)\right\|_{L^{p}(\Omega)}+\left\|\phi_{n}(t)-\phi_{\mathbf{P}}(t)\right\|_{L^{p}(\Omega)}\right)\|\overline{\mathbf{P}}\|_{L^{\frac{p}{p-2}}(\Omega)} \\
\leq & C K_{\mathbf{P}}^{2} C_{\mathbf{P}}\left\|l_{2}^{n}(t)-l_{2}(t)\right\|_{\mathbb{W}_{p}^{*}}\|\overline{\mathbf{P}}\|_{W^{1, p^{\prime}}(\Omega)}
\end{aligned}
$$

and

$$
\begin{aligned}
& \left|\mathcal{S}_{3}\left(\phi_{n}(t)\right)-\mathcal{S}_{3}\left(\phi_{\mathbf{P}}(t)\right)\right| \\
\leq & C\left\|\nabla \phi_{n}(t)-\nabla \phi_{\mathbf{P}}(t)\right\|_{L^{p}(\Omega)}\|\overline{\mathbf{P}}\|_{L^{\frac{p}{p-2}}(\Omega)} \\
\leq & C C_{\mathbf{P}}\left\|l_{2}^{n}(t)-l_{2}(t)\right\|_{\mathbb{W}_{p}^{*}}\|\overline{\mathbf{P}}\|_{W^{1, p^{\prime}}(\Omega)} .
\end{aligned}
$$

From (4.17) we know that the norms $\left\|l_{2}^{n}(t)\right\|_{\mathbb{W}_{*}^{*}}$ are uniformly bounded for all $n \in \mathbb{N}$, thus (4.32) to (4.34) and (4.17) immediately imply (4.19). This completes the proof of Step 1.

\section{Step 2: validity of condition (S)}

We recall the condition (S): Let $M>0$ be an arbitrary positive number. Then we need to show that there exists some $h_{M} \in L^{\tau}(0, T)$, such that for all $\mathbf{P}_{1}, \mathbf{P}_{2} \in$ $(Y, X)_{\frac{1}{\tau}, \tau}$ with

$$
\max \left\{\left\|\mathbf{P}_{1}\right\|_{(Y, X)_{\frac{1}{\tau}, \tau}},\left\|\mathbf{P}_{2}\right\|_{(Y, X)_{\frac{1}{\tau}, \tau}}\right\} \leq M
$$

we have

$$
\left\|S\left(t, \mathbf{P}_{1}\right)-S\left(t, \mathbf{P}_{2}\right)\right\|_{X} \leq h_{M}(t)\left\|\mathbf{P}_{1}-\mathbf{P}_{2}\right\|_{(Y, X)_{\frac{1}{\tau}, \tau}}
$$


for a.a. $t \in(0, T)$. From Lemma 4.4 we deduce that the set

$$
\mathcal{V}:=\left\{\mathbf{P} \in(Y, X)_{\frac{1}{\tau}, \tau}:\|\mathbf{P}\|_{(Y, X)_{\frac{1}{\tau}, \tau}} \leq M\right\}
$$

is a bounded subset of $\left(L^{\infty}(\Omega)\right)^{d}$. We may then assume that $\|\mathbf{P}\|_{L^{\infty}(\Omega)} \leq M^{\prime}$ for all $\mathbf{P} \in \mathcal{V}$ with some $M^{\prime}>0$ depending on $M$. Using Assumption (A2) we can find some $C_{M} \in\left(\max \left\{1, M^{\prime}\right\}, \infty\right)$ such that

$$
\begin{aligned}
\sup _{\boldsymbol{p} \in \mathbb{R}^{d},|\boldsymbol{p}| \leq M^{\prime}}\left\{\operatorname{Lip}_{D_{p} \mathbb{C}}, \operatorname{Lip}_{D_{\boldsymbol{p}} \mathbf{e}}, \operatorname{Lip}_{D_{\boldsymbol{p}} \varepsilon^{0}}, \operatorname{Lip}_{D_{\boldsymbol{p}} \boldsymbol{\epsilon}}, \operatorname{Lip}_{D_{\boldsymbol{p}} \omega}\right. \\
|\mathbb{C}(\boldsymbol{p})|,|\mathbf{e}(\boldsymbol{p})|,\left|\varepsilon^{0}(\boldsymbol{p})\right|,|\boldsymbol{\epsilon}(\boldsymbol{p})|,|\omega(\boldsymbol{p})| \\
\left.\left|D_{\boldsymbol{p}} \mathbb{C}(\boldsymbol{p})\right|,\left|D_{\boldsymbol{p}} \mathbf{e}(\boldsymbol{p})\right|,\left|D_{\boldsymbol{p}} \varepsilon^{0}(\boldsymbol{p})\right|,\left|D_{\boldsymbol{p}} \boldsymbol{\epsilon}(\boldsymbol{p})\right|,\left|D_{\boldsymbol{p}} \omega(\boldsymbol{p})\right|\right\} \leq C_{M},
\end{aligned}
$$

where for a Lipschitz function $f$ defined on $\mathbb{R}^{d}, \operatorname{Lip}_{f}$ denotes its Lipschitz constant on the closed ball $\bar{B}_{M^{\prime}}(0) \subset \mathbb{R}^{d}$. Let $\mathbf{P}_{1}, \mathbf{P}_{2} \in \mathcal{V}$. Consider the difference

$$
\begin{aligned}
& S\left(t, \mathbf{P}_{1}\right)-S\left(t, \mathbf{P}_{2}\right) \\
= & -\left(D_{\mathbf{P}} H\left(\varepsilon\left(\mathbf{u}_{\mathbf{P}_{1}}(t)\right), \nabla \phi_{\mathbf{P}_{1}}(t), \mathbf{P}_{1}\right)-D \mathbf{P} H\left(\varepsilon\left(\mathbf{u}_{\mathbf{P}_{2}}(t)\right), \nabla \phi_{\mathbf{P}_{2}}(t), \mathbf{P}_{2}\right)\right) \\
& -\left(D_{\mathbf{P}} \omega\left(\mathbf{P}_{1}\right)-D_{\mathbf{P}} \omega\left(\mathbf{P}_{2}\right)\right) \\
= & : \mathcal{I}_{1}+\mathcal{I}_{2} .
\end{aligned}
$$

To estimate $\mathcal{I}_{2}$, we obtain that

$$
\begin{aligned}
& \int_{\Omega}\left|D_{\mathbf{P}} \omega\left(\mathbf{P}_{1}\right)-D_{\mathbf{P}} \omega\left(\mathbf{P}_{2}\right)\right| \cdot|\overline{\mathbf{P}}| d x \\
\leq & C\left\|D_{\mathbf{P}} \omega\left(\mathbf{P}_{1}\right)-D_{\mathbf{P}} \omega\left(\mathbf{P}_{2}\right)\right\|_{L^{p}(\Omega)}\|\overline{\mathbf{P}}\|_{L^{\frac{p}{p^{-2}}}(\Omega)} \\
\leq & C C_{M}\left\|\mathbf{P}_{1}-\mathbf{P}_{2}\right\|_{L^{p}(\Omega)}\|\overline{\mathbf{P}}\|_{W^{1, p^{\prime}}(\Omega)} \\
\leq & C C_{M}\left\|\mathbf{P}_{1}-\mathbf{P}_{2}\right\|_{L^{\infty}(\Omega)}\|\overline{\mathbf{P}}\|_{W^{1, p^{\prime}}(\Omega)} \\
\leq & C C_{M}\left\|\mathbf{P}_{1}-\mathbf{P}_{2}\right\|_{(Y, X)_{\frac{1}{\tau}, \tau}}\|\overline{\mathbf{P}}\|_{W^{1, p^{\prime}}(\Omega)}
\end{aligned}
$$

from which we infer that

$$
\left\|\mathcal{I}_{2}\right\|_{X} \leq C C_{M}\left\|\mathbf{P}_{1}-\mathbf{P}_{2}\right\|_{(Y, X)_{\frac{1}{\tau}, \tau}} .
$$

To estimate $\mathcal{I}_{1}$, we use (4.20) to obtain that

$$
\begin{aligned}
& \int_{\Omega}\left(D_{\mathbf{P}} H\left(\varepsilon\left(\mathbf{u}_{\mathbf{P}_{1}}(t)\right), \nabla \phi_{\mathbf{P}_{1}}(t), \mathbf{P}_{1}\right)-D_{\mathbf{P}} H\left(\varepsilon\left(\mathbf{u}_{\mathbf{P}_{2}}(t)\right), \nabla \phi_{\mathbf{P}_{2}}(t), \mathbf{P}_{2}\right)\right) \cdot \overline{\mathbf{P}} d x \\
= & \left(\mathcal{S}_{1}\left(\mathbf{u}_{\mathbf{P}_{1}}(t), \phi_{\mathbf{P}_{1}}(t), \mathbf{P}_{1}\right)-\mathcal{S}_{1}\left(\mathbf{u}_{\mathbf{P}_{2}}(t), \phi_{\mathbf{P}_{2}}(t), \mathbf{P}_{2}\right)\right) \\
+ & \left(\mathcal{S}_{2}\left(\mathbf{u}_{\mathbf{P}_{1}}(t), \phi_{\mathbf{P}_{1}}(t), \mathbf{P}_{1}\right)-\mathcal{S}_{2}\left(\mathbf{u}_{\mathbf{P}_{2}}(t), \phi_{\mathbf{P}_{2}}(t), \mathbf{P}_{2}\right)\right) \\
+ & \left(\mathcal{S}_{3}\left(\phi_{\mathbf{P}_{1}}(t)\right)-\mathcal{S}_{3}\left(\phi_{\mathbf{P}_{2}}(t)\right)\right) .
\end{aligned}
$$

From the definition of $\left(\mathbf{u}_{\mathbf{P}_{j}}, \phi_{\mathbf{P}_{j}}\right)$ with $j=1,2$ we know that

$$
\mathrm{L}_{\mathbf{P}_{j}}\left(\mathbf{u}_{\mathbf{P}_{i}}(t), \phi_{\mathbf{P}_{j}}(t)\right)=\ell_{1}\left(\mathbf{P}_{j}\right)+\ell_{2}(t)
$$


and

$$
\begin{aligned}
& \mathrm{L}_{\mathbf{P}_{1}}\left(\mathbf{u}_{\mathbf{P}_{1}}(t)-\mathbf{u}_{\mathbf{P}_{2}}(t), \phi_{\mathbf{P}_{1}}(t)-\phi_{\mathbf{P}_{2}}(t)\right) \\
= & \ell_{1}\left(\mathbf{P}_{1}\right)-\ell_{1}\left(\mathbf{P}_{2}\right)-\left(\left[\mathrm{L}_{\mathbf{P}_{1}}-\mathrm{L}_{\mathbf{P}_{2}}\right]\left(\mathbf{u}_{\mathbf{P}_{2}}(t), \phi_{\mathbf{P}_{2}}(t)\right)\right) .
\end{aligned}
$$

From (2.8) and (4.12) it follows

$$
\begin{aligned}
\left\|\ell_{1}\left(\mathbf{P}_{j}\right)\right\|_{\mathbb{W}_{p}^{*}} & \leq C C_{M}^{2}, \\
\left\|\ell_{1}\left(\mathbf{P}_{1}\right)-\ell_{1}\left(\mathbf{P}_{2}\right)\right\|_{\mathbb{W}_{p}^{*}} & \leq C C_{M}^{2}\left\|\mathbf{P}_{1}-\mathbf{P}_{2}\right\|_{L^{\infty}(\Omega)}
\end{aligned}
$$

and

$$
\begin{aligned}
&\left\|\left[\mathrm{L}_{\mathbf{P}_{1}}-\mathrm{L}_{\mathbf{P}_{2}}\right]\left(\mathbf{u}_{\mathbf{P}_{2}}(t), \phi_{\mathbf{P}_{2}}(t)\right)\right\|_{\mathbb{W}_{p}^{*}} \\
& \leq C C_{M}\left\|\mathbf{P}_{1}-\mathbf{P}_{2}\right\|_{L^{\infty}(\Omega)}\left\|\left(\mathbf{u}_{\mathbf{P}_{2}}(t), \phi_{\mathbf{P}_{2}}(t)\right)\right\|_{\mathbb{W}_{p}} .
\end{aligned}
$$

Applying (4.10) on (4.40) and (4.41) and using (4.42) and (4.43) we obtain that

$$
\left\|\left(\mathbf{u}_{\mathbf{P}_{j}}(t), \phi_{\mathbf{P}_{j}}(t)\right)\right\|_{\mathbb{W}_{p}} \leq C C^{*} C_{M}^{2}\left(1+\left\|l_{2}(t)\right\|_{\mathbb{W}_{p}^{*}}\right)
$$

with $C^{*}$ the number from Lemma 4.6 corresponding to the set $\mathcal{V}$ (notice here that Lemma 4.6 is applicable, since $\mathcal{V}$ is due to Lemma 4.10 a closed and bounded subset of some Hölder space, and Hölder spaces are compactly embedded into the space of continuous functions), and

$$
\begin{aligned}
& \left\|\left(\mathbf{u}_{\mathbf{P}_{1}}(t)-\mathbf{u}_{\mathbf{P}_{2}}(t), \phi_{\mathbf{P}_{1}}(t)-\phi_{\mathbf{P}_{2}}(t)\right)\right\|_{\mathbb{W}_{p}} \\
\leq & C C^{*} C_{M}^{2}\left\|\mathbf{P}_{1}-\mathbf{P}_{2}\right\|_{L^{\infty}(\Omega)}+C C^{*} C_{M}\left\|\mathbf{P}_{1}-\mathbf{P}_{2}\right\|_{L^{\infty}(\Omega)}\left\|\left(\mathbf{u}_{\mathbf{P}_{2}}(t), \phi_{\mathbf{P}_{2}}(t)\right)\right\|_{\mathbb{W}_{p}} \\
\leq & C C^{*} C_{M}^{2}\left\|\mathbf{P}_{1}-\mathbf{P}_{2}\right\|_{L^{\infty}(\Omega)}\left(1+\left\|\left(\mathbf{u}_{\mathbf{P}_{2}}(t), \phi_{\mathbf{P}_{2}}(t)\right)\right\|_{\mathbb{W}_{p}}\right) \\
\stackrel{(4.45)}{\leq} & C C^{*} C_{M}^{2}\left\|\mathbf{P}_{1}-\mathbf{P}_{2}\right\|_{L^{\infty}(\Omega)}\left(C C^{*} C_{M}^{2}\left(1+\left\|l_{2}(t)\right\|_{\mathbb{W}_{p}^{*}}\right)\right) \\
\leq & C\left(C^{*}\right)^{2} C_{M}^{4}\left(1+\left\|l_{2}(t)\right\|_{\mathbb{W}_{p}^{*}}\right)\left\|\mathbf{P}_{1}-\mathbf{P}_{2}\right\|_{L^{\infty}(\Omega)} .
\end{aligned}
$$

Combining with telescoping technique, (4.45) and (4.46) yield

$$
\begin{aligned}
& \left|\mathcal{S}_{1}\left(\mathbf{u}_{\mathbf{P}_{1}}(t), \phi_{\mathbf{P}_{1}}(t), \mathbf{P}_{1}\right)-\mathcal{S}_{1}\left(\mathbf{u}_{\mathbf{P}_{2}}(t), \phi_{\mathbf{P}_{2}}(t), \mathbf{P}_{2}\right)\right| \\
\leq & \int_{\Omega} C C_{M}^{3}\left|\mathbf{P}_{1}-\mathbf{P}_{2}\right|\left(1+\left|\varepsilon\left(\mathbf{u}_{\mathbf{P}_{1}}(t)\right)\right|^{2}+\left|\nabla \phi_{\mathbf{P}_{1}}(t)\right|^{2}\right)|\overline{\mathbf{P}}| d x \\
+ & \int_{\Omega} C C_{M}^{3}\left(\left|\varepsilon\left(\mathbf{u}_{\mathbf{P}_{1}}(t)\right)-\varepsilon\left(\mathbf{u}_{\mathbf{P}_{2}}(t)\right)\right|+\left|\nabla \phi_{\mathbf{P}_{1}}(t)-\nabla \phi_{\mathbf{P}_{2}}(t)\right|+\left|\mathbf{P}_{1}-\mathbf{P}_{2}\right|\right) \\
& \cdot\left(1+\sum_{j=1}^{2}\left(\left|\varepsilon\left(\mathbf{u}_{\mathbf{P}_{j}}(t)\right)\right|+\left|\nabla \phi_{\mathbf{P}_{j}}(t)\right|\right)\right)|\overline{\mathbf{P}}| d x \\
\leq & C C_{M}^{3}\left\|\mathbf{P}_{1}-\mathbf{P}_{2}\right\|_{L^{p}(\Omega)}\left(1+\left\|\varepsilon\left(\mathbf{u}_{\mathbf{P}_{1}}(t)\right)\right\|_{L^{p}(\Omega)}^{2}+\left\|\nabla \phi_{\mathbf{P}_{1}}(t)\right\|_{L^{p}(\Omega)}^{2}\right)\|\overline{\mathbf{P}}\|_{L^{\frac{p}{p-2}}(\Omega)} \\
+ & C C_{M}^{3}\|\overline{\mathbf{P}}\|_{L^{\frac{p}{p-2}}(\Omega)}\left(\left\|\varepsilon\left(\mathbf{u}_{\mathbf{P}_{1}}(t)\right)-\varepsilon\left(\mathbf{u}_{\mathbf{P}_{2}}(t)\right)\right\|_{L^{p}(\Omega)}+\left\|\mathbf{P}_{1}-\mathbf{P}_{2}\right\|_{L^{p}(\Omega)}\right.
\end{aligned}
$$




$$
\begin{aligned}
& \left.+\left\|\nabla \phi_{\mathbf{P}_{1}}(t)-\nabla \phi_{\mathbf{P}_{2}}(t)\right\|_{L^{p}(\Omega)}\right) \cdot\left(1+\sum_{j=1}^{2}\left(\left\|\varepsilon\left(\mathbf{u}_{\mathbf{P}_{j}}(t)\right)\right\|_{L^{p}(\Omega)}+\left\|\nabla \phi_{\mathbf{P}_{j}}(t)\right\|_{L^{p}(\Omega)}\right)\right) \\
& \leq C C_{M}^{3}\left(\left\|\left(\mathbf{u}_{\mathbf{P}_{1}}(t)-\mathbf{u}_{\mathbf{P}_{2}}(t), \phi_{\mathbf{P}_{1}}(t)-\phi_{\mathbf{P}_{2}}(t)\right)\right\|_{\mathbb{W}_{p}}+\left\|\mathbf{P}_{1}-\mathbf{P}_{2}\right\|_{L^{\infty}(\Omega)}\right) \\
& \cdot\left(1+\sum_{j=1}^{2}\left\|\left(\mathbf{u}_{\mathbf{P}_{j}}(t), \phi_{\mathbf{P}_{j}}(t)\right)\right\| \|_{\mathbb{W}_{p}}\right)\|\overline{\mathbf{P}}\|_{W^{1, p^{\prime}}(\Omega)} \\
& \leq C\left(C^{*}\right)^{3} C_{M}^{9}\left(1+\left\|l_{2}(t)\right\|_{\mathbb{W}_{p}^{*}}^{2}\right)\left\|\mathbf{P}_{1}-\mathbf{P}_{2}\right\|_{L^{\infty}(\Omega)}\|\overline{\mathbf{P}}\|_{W^{1, p^{\prime}}(\Omega)} .
\end{aligned}
$$

In the same manner, we obtain that

$$
\begin{aligned}
& \left|\mathcal{S}_{2}\left(\mathbf{u}_{\mathbf{P}_{1}}(t), \phi_{\mathbf{P}_{1}}(t), \mathbf{P}_{1}\right)-\mathcal{S}_{2}\left(\mathbf{u}_{\mathbf{P}_{2}}(t), \phi_{\mathbf{P}_{2}}(t), \mathbf{P}_{2}\right)\right| \\
\leq & \int_{\Omega} C C_{M}^{3}\left|\mathbf{P}_{1}-\mathbf{P}_{2}\right|\left(1+\left|\varepsilon\left(\mathbf{u}_{\mathbf{P}_{1}}(t)\right)\right|+\left|\nabla \phi_{\mathbf{P}_{1}}(t)\right|\right)|\overline{\mathbf{P}}| d x \\
+ & \int_{\Omega} C_{M}^{3}\left(\left|\varepsilon\left(\mathbf{u}_{\mathbf{P}_{1}}(t)\right)-\varepsilon\left(\mathbf{u}_{\mathbf{P}_{2}}(t)\right)\right|+\left|\nabla \phi_{\mathbf{P}_{1}}(t)-\nabla \phi_{\mathbf{P}_{2}}(t)\right|+\left|\mathbf{P}_{1}-\mathbf{P}_{2}\right|\right)|\overline{\mathbf{P}}| d x \\
\leq & C C_{M}^{3}\left\|\mathbf{P}_{1}-\mathbf{P}_{2}\right\|_{L^{p}(\Omega)}\left(1+\left\|\varepsilon\left(\mathbf{u}_{\mathbf{P}_{1}}(t)\right)\right\|_{L^{p}(\Omega)}+\left\|\nabla \phi_{\mathbf{P}_{1}}(t)\right\|_{L^{p}(\Omega)}\right)\|\overline{\mathbf{P}}\|_{L^{\frac{p}{p-2}}(\Omega)} \\
+ & C C_{M}^{3}\left(\left\|\varepsilon\left(\mathbf{u}_{\mathbf{P}_{1}}(t)\right)-\varepsilon\left(\mathbf{u}_{\mathbf{P}_{2}}(t)\right)\right\|_{L^{p}(\Omega)}+\left\|\nabla \phi_{\mathbf{P}_{1}}(t)-\nabla \phi_{\mathbf{P}_{2}}(t)\right\|_{L^{p}(\Omega)}\right. \\
+ & \left.\left\|\mathbf{P}_{1}-\mathbf{P}_{2}\right\|_{L^{p}(\Omega)}\right)\|\overline{\mathbf{P}}\|_{L^{\frac{p}{p-2}}(\Omega)} \\
\leq & C C^{*} C_{M}^{5}\left(1+\left\|l_{2}(t)\right\|_{\mathbb{W}_{p}^{*}}\right)\left\|\mathbf{P}_{1}-\mathbf{P}_{2}\right\|_{L^{\infty}(\Omega)}\|\overline{\mathbf{P}}\|_{W^{1, p^{\prime}}(\Omega)} \\
+ & C\left(C^{*}\right)^{2} C_{M}^{7}\left(1+\left\|l_{2}(t)\right\|_{\mathbb{W}_{p}^{*}}\right)\left\|\mathbf{P}_{1}-\mathbf{P}_{2}\right\|_{L^{\infty}(\Omega)}\|\overline{\mathbf{P}}\|_{W^{1, p^{\prime}}(\Omega)} \\
\leq & C\left(C^{*}\right)^{3} C_{M}^{9}\left(1+\left\|l_{2}(t)\right\|_{\mathbb{W}_{p}^{*}}^{2}\right)\left\|\mathbf{P}_{1}-\mathbf{P}_{2}\right\|_{L^{\infty}(\Omega)}\|\overline{\mathbf{P}}\|_{W^{1, p^{\prime}}(\Omega)}
\end{aligned}
$$

and

$$
\begin{aligned}
& \left|\mathcal{S}_{3}\left(\phi_{\mathbf{P}_{1}}(t)\right)-\mathcal{S}_{3}\left(\phi_{\mathbf{P}_{2}}(t)\right)\right| \\
\leq & \int_{\Omega}\left|\nabla \phi_{\mathbf{P}_{1}}(t)-\nabla \phi_{\mathbf{P}_{2}}(t)\right| \cdot|\overline{\mathbf{P}}| d x \\
\leq & C\left\|\nabla \phi_{\mathbf{P}_{1}}(t)-\nabla \phi_{\mathbf{P}_{2}}(t)\right\|_{L^{p}(\Omega)}\|\overline{\mathbf{P}}\|_{L^{\frac{p}{p-2}}(\Omega)} \\
\leq & C\left(C^{*}\right)^{2} C_{M}^{4}\left(1+\left\|l_{2}(t)\right\|_{\mathbb{W}_{p}^{*}}\right)\left\|\mathbf{P}_{1}-\mathbf{P}_{2}\right\|_{L^{\infty}(\Omega)}\|\overline{\mathbf{P}}\|_{W^{1, p^{\prime}}(\Omega)} \\
\leq & C\left(C^{*}\right)^{3} C_{M}^{9}\left(1+\left\|l_{2}(t)\right\|_{\mathbb{W}_{p}^{*}}^{2}\right)\left\|\mathbf{P}_{1}-\mathbf{P}_{2}\right\|_{L^{\infty}(\Omega)}\|\overline{\mathbf{P}}\|_{W^{1, p^{\prime}}(\Omega)}
\end{aligned}
$$

for some sufficiently large $C$. Hence

$$
\begin{aligned}
\left\|\mathcal{I}_{1}\right\|_{X} & \leq C\left(C^{*}\right)^{3} C_{M}^{9}\left(1+\left\|l_{2}(t)\right\|_{\mathbb{W}_{p}^{*}}^{2}\right)\left\|\mathbf{P}_{1}-\mathbf{P}_{2}\right\|_{L^{\infty}(\Omega)} \\
& \leq C\left(C^{*}\right)^{3} C_{M}^{9}\left(1+\left\|l_{2}(t)\right\|_{\mathbb{W}_{p}^{*}}^{2}\right)\left\|\mathbf{P}_{1}-\mathbf{P}_{2}\right\|_{(Y, X)_{\frac{1}{\tau}, \tau}} .
\end{aligned}
$$

From Assumption (A4) it follows that $\left(1+\left\|l_{2}(t)\right\|_{\mathbb{W}_{p}^{*}}^{2}\right) \in L^{\tau}(0, T)$, thus setting

$$
h_{M}(t)=C\left(C^{*}\right)^{3} C_{M}^{9}\left(1+\left\|l_{2}(t)\right\|_{\mathbb{W}_{p}^{*}}^{2}\right)+C C_{M}
$$


with $C C_{M}$ from (4.38), completes the proof of Step 2.

Step 3: verification of $S(\cdot, 0) \in L^{\tau}(0, T ; X)$

Recall that

$$
S(t, 0)=-D_{\mathbf{P}} H\left(\varepsilon\left(\mathbf{u}_{0}(t)\right), \nabla \phi_{0}(t), 0\right)-D_{\mathbf{P}} \omega(0)+\ell_{3}(t) .
$$

From Assumption (A4) we know that $\ell_{3} \in L^{\tau}(0, T ; X)$; Since $-D_{\mathbf{P}} \omega(0)$ is a constant, it is also an element of $L^{\tau}(0, T ; X)$; Finally, by setting $(\mathbf{u}, \phi)=$ $\left(\mathbf{u}_{0}(t), \phi_{0}(t)\right)$ in (4.21) and (4.23) and using (4.29) with $\mathbf{P}=0$ and Assumption (A4) we conclude that

$$
-D_{\mathbf{P}} H\left(\varepsilon\left(\mathbf{u}_{0}(t)\right), \nabla \phi_{0}(t), 0\right) \in L^{\tau}(0, T ; X) .
$$

Hence we infer that $S(\cdot, 0) \in L^{\tau}(0, T ; X)$.

Sum up all, we see that all conditions of Theorem 4.3 are satisfied and one obtains from Theorem 4.3 a unique local in time solution

$$
\mathbf{P} \in W^{1, r}\left(0, \hat{T} ;\left(W_{\Gamma_{\mathbf{P}}}^{-1, p}(\Omega)\right)^{d}\right) \cap L^{r}\left(0, \hat{T} ;\left(W_{\Gamma_{\mathbf{P}}}^{1, p}(\Omega)\right)^{d}\right)
$$

of the equation

$$
\mathbf{P}^{\prime}(t)-\Delta \mathbf{P}(t)=S(t, \mathbf{P}(t))
$$

on the maximal time interval $(0, \hat{T})$ for some $\hat{T} \in(0, T]$. For the given pair $(t, \mathbf{P}(t))$ one obtains the unique weak solution $(\mathbf{u}(t), \phi(t))$ of (2.1a), that is

$$
L_{\mathbf{P}(t)}(\mathbf{u}(t), \phi(t))=\ell(t, \mathbf{P}(t)) .
$$

From Proposition 4.5 we find some positive constant $M^{\prime \prime}$ and some $\delta \in(0,1)$ such that

$$
\max _{t \in[0, T]}\|\mathbf{P}(t)\|_{C^{\delta}(\bar{\Omega})} \leq M^{\prime \prime} .
$$

We then define the set $\mathcal{M}$ in Lemma 4.6 by

$$
\mathcal{M}:=\left\{\mathbf{P} \in\left(C^{\delta}(\bar{\Omega})\right)^{d}:\|\mathbf{P}\|_{C^{\delta}(\bar{\Omega})} \leq M^{\prime \prime}\right\}
$$

and denote by $\left(C^{*}\right)^{\prime}$ the corresponding uniform bound of inverse norm $\mathrm{L}_{\mathbf{P}}^{-1}$ given in Lemma 4.6. Using the definition of $\ell_{1}(\mathbf{P})$ given in (2.8), together with Assumption (A2) and (4.52), it follows immediately that

$$
\max _{t \in[0, T]}\left\|\ell_{1}(\mathbf{P}(t))\right\|_{\mathbb{W}_{p}^{*}} \leq C_{M^{\prime \prime}}
$$

for some positive constant $C_{M^{\prime \prime}}$ which only depends on $M^{\prime \prime}$. Using (4.10) we infer that

$$
\|(\mathbf{u}(t), \phi(t))\|_{\mathbb{W}_{p}} \leq\left(C^{*}\right)^{\prime}\left(C_{M^{\prime \prime}}+\left\|\ell_{2}(t)\right\|_{\mathbb{W}_{p}^{*}}\right) .
$$

On the other hand, the Bochner-measurability of $(\mathbf{u}, \phi):[0, T] \rightarrow \mathbb{W}_{p}$ can be similarly shown as by Step 1 given previously, we omit the details here. Hence the claimed regularity of $(\mathbf{u}, \phi)$ given in Definition 2.1 follows immediately from Assumption (A4) and (4.53). This completes the desired proof of Theorem 2.3 


\section{Proof of the elliptic regularity results}

\subsection{Proof of Theorem 2.4}

In this section we give the proof of Theorem 2.4. The main ingredient is Theorem 6.2 from 18 , which will be restated in Theorem 5.2 below. To introduce the result we firstly fix some notation. Let $m \in \mathbb{N}$. For $1 \leq i \leq m$ and $\Gamma_{i} \subset \partial \Omega$ we define the space

$$
\mathbb{W}_{\Gamma}^{1, p}:=\prod_{i=1}^{m} W_{\Gamma_{i}}^{1, p}(\Omega) .
$$

The dual space of $\mathbb{W}_{\Gamma}^{1, p^{\prime}}$ is denoted by $\mathbb{W}_{\Gamma}^{-1, p}$. For $\mathbb{A} \in L^{\infty}\left(\Omega, \operatorname{Lin}\left(\mathbb{R}^{m} \times\right.\right.$ $\left.\mathbb{R}^{m d}, \mathbb{R}^{m} \times \mathbb{R}^{m d}\right)$ ) we define the operator $\mathcal{A}$ by

$$
\left\langle\mathcal{A}\left(\mathbf{v}_{1}\right), \mathbf{v}_{2}\right\rangle:=\int_{\Omega} \mathbb{A}\left(\begin{array}{c}
\mathbf{v}_{1} \\
\nabla \mathbf{v}_{1}
\end{array}\right):\left(\begin{array}{c}
\mathbf{v}_{2} \\
\nabla \mathbf{v}_{2}
\end{array}\right) d x
$$

for $\mathbf{v}_{1}, \mathbf{v}_{2} \in \mathbb{W}_{\Gamma}^{1,2}$. Additionally we will also need the following assumption:

Assumption 5.1. Let $\Gamma \subset \partial \Omega$ be closed. There exists a linear, continuous extension operator $\mathbb{E}: W_{\Gamma}^{1,1}(\Omega) \rightarrow W_{\Gamma}^{1,1}\left(\mathbb{R}^{d}\right)$ which simultaneously defines a continuous extension operator $\mathbb{E}: W_{\Gamma}^{1, p}(\Omega) \rightarrow W_{\Gamma}^{1, p}\left(\mathbb{R}^{d}\right)$ for all $p \in(1, \infty)$.

Having all the preliminaries we are now ready to state Theorem 5.2

Theorem 5.2 ([18, Thm. 6.2]). Let $\Omega \subset \mathbb{R}^{d}, d \geq 2$, be a bounded domain. Let all $\Gamma_{i} \subset \partial \Omega$ be $(d-1)$-sets and let the Assumption 5.1 be satisfied for all $\Gamma_{i}$. Furthermore, assume that there exists a positive constant $\eta$ such that

$$
\langle\mathcal{A}(\mathbf{v}), \mathbf{v}\rangle \geq \eta\|\mathbf{v}\|_{H^{1}(\Omega)}
$$

for all $\mathbf{v} \in \mathbb{W}_{\Gamma}^{1,2}$. Then there exists some $p^{*}>2$ such that for all $p \in\left[2, p^{*}\right]$, the operator $\mathrm{L}_{\mathcal{A}}: \mathbf{v} \mapsto\langle\mathcal{A}(\mathbf{v}), \cdot\rangle$ defines a topological isomorphism from $\mathbb{W}_{\Gamma}^{1, p}$ to $\mathbb{W}_{\Gamma}^{-1, p}$. In particular, the norm $\left\|\mathrm{L}_{\mathcal{A}}^{-1}\right\|_{L\left(\mathbb{W}_{\Gamma}^{-1, p}, \mathbb{W}_{\Gamma}^{1, p}\right)}$ depends only on $\eta$ and $\|\mathbb{A}\|_{L^{\infty}(\Omega)}$ and is uniform for all $p \in\left[2, p^{*}\right]$.

We now give the proof of Theorem 2.4.

Proof of Theorem 2.4. To shed light on the connection, we set the space $\mathbb{W}_{\Gamma}^{1, p}$ by

$$
\mathbb{W}_{\Gamma}^{1, p}=\mathbb{W}_{p}
$$

and the operator $\mathcal{A}$ and tensor $\mathbb{A}$ by the following implicit form

$$
\begin{aligned}
& \left\langle\mathcal{A}\left(\left(\mathbf{u}_{1}, \phi_{1}\right)\right),\left(\mathbf{u}_{2}, \phi_{2}\right)\right\rangle \\
= & \int_{\Omega} \mathbb{A}\left(\begin{array}{c}
\mathbf{u}_{1} \\
\phi_{1} \\
\nabla \mathbf{u}_{1} \\
\nabla \phi_{1}
\end{array}\right):\left(\begin{array}{c}
\mathbf{u}_{2} \\
\phi_{2} \\
\nabla \mathbf{u}_{2} \\
\nabla \phi_{2}
\end{array}\right) d x \\
= & \mathrm{L}_{\mathbf{P}}\left(\mathbf{u}_{1}, \phi_{1}\right)\left[\left(\mathbf{u}_{2}, \phi_{2}\right)\right] .
\end{aligned}
$$


It suffices to show that the conditions in Theorem 5.2 are satisfied. Firstly we point out that the Assumption (B1) already implies Assumption 5.1, see [18, Thm. 4.15]. On the other hand, from Assumption (B2) we know that the tensor $\mathbb{A}$ is of class $L^{\infty}$. It is therefore only left to show the coercivity condition (5.1). From Assumption (B3). Poincaré's and Korn's inequalities and the fact that the antidiagonals of the tensor $\mathbb{B}(\mathbf{P})$ given by (1.7) are composed by $\mathbf{e}(\mathbf{P})^{T}$ and $-\mathbf{e}(\mathbf{P})$ we obtain that

$$
\begin{aligned}
& \mathrm{L}_{\mathbf{P}}(\mathbf{u}, \phi)[(\mathbf{u}, \phi)] \\
= & \int_{\Omega} \mathbb{C}(\mathbf{P}) \varepsilon(\mathbf{u}): \varepsilon(\mathbf{u})+\boldsymbol{\epsilon}(\mathbf{P}) \nabla \phi \cdot \nabla \phi d x \\
\geq & \alpha\left(\|\varepsilon(\mathbf{u})\|_{L^{2}(\Omega)}^{2}+\|\nabla \phi\|_{L^{2}(\Omega)}^{2}\right) \\
\geq & \alpha \min \left\{c_{P}, c_{K}\right\}\left(\|\mathbf{u}\|_{H^{1}(\Omega)}^{2}+\|\phi\|_{H^{1}(\Omega)}^{2}\right)
\end{aligned}
$$

with $\alpha>0$ from Assumption (B3) and $c_{P}, c_{K}>0$ the corresponding Poincaré's and Korn's constants, and (5.1) is fulfilled. This completes the desired proof.

\subsection{Proof of Theorem 2.6}

In this section we prove Theorem 2.6. In fact, we will prove the following stronger version of Theorem 2.6, in the sense that $p$ can be arbitrarily chosen from the interval $[2, \infty)$ and $\mathbf{P}$ is merely assumed to be an element of $(C(\bar{\Omega}))^{d}$.

Theorem 5.3. Let the Assumptions $(\mathrm{C1})$ and $(\mathrm{C2})$ be satisfied. Let $\mathbf{P} \in$ $(C(\bar{\Omega}))^{d}$ and $p \in[2, \infty)$. Then the differential operator $\mathrm{L}_{\mathbf{P}}$ defines a topological isomorphism from $\left(W_{0}^{1, p}(\Omega)\right)^{d}$ to $\left(W^{-1, p}(\Omega)\right)^{d}$.

Proof. Since $\mathbf{P}$ is continuous on $\bar{\Omega}$ and $\alpha$ is continuous and non-negative on $\mathbb{R}^{d}$ due to Assumption $(\mathrm{C} 2)$, we infer that $\alpha(\mathbf{P}(\cdot))$ is continuous and non-negative on the compact set $\bar{\Omega}$, thus also bounded below by some positive constant on $\bar{\Omega}$. We can hence define

$$
\alpha_{\mathbf{P}}:=\min \{\alpha(\mathbf{P}(x)): x \in \bar{\Omega}\}>0 .
$$

From Assumption $(\mathrm{C} 2)$ and Poincaré's and Korn's inequalities we obtain that

$$
\begin{aligned}
& \mathrm{L}_{\mathbf{P}}(\mathbf{u}, \phi)[(\mathbf{u}, \phi)] \\
= & \int_{\Omega} \mathbb{C}(\mathbf{P}) \varepsilon(\mathbf{u}): \varepsilon(\mathbf{u})+\boldsymbol{\epsilon}(\mathbf{P}) \nabla \phi \cdot \nabla \phi d x \\
\geq & \int_{\Omega} \alpha(\mathbf{P})\left(|\varepsilon(\mathbf{u})|^{2}+|\nabla \phi|^{2}\right) d x \\
\geq & \alpha_{\mathbf{P}}\left(\|\boldsymbol{\varepsilon}(\mathbf{u})\|_{L^{2}(\Omega)}^{2}+\|\nabla \phi\|_{L^{2}(\Omega)}^{2}\right) \\
\geq & \alpha_{\mathbf{P}} \min \left\{c_{P}, c_{K}\right\}\left(\|\mathbf{u}\|_{H^{1}(\Omega)}^{2}+\|\phi\|_{H^{1}(\Omega)}^{2}\right)
\end{aligned}
$$

and therefore $L_{\mathbf{P}}$ is coercive. Since $\mathbb{B}$ is a continuous function on $\mathbb{R}^{d}$ due to Assumption $(\mathrm{C} 2)$ and $\mathbf{P} \in(C(\bar{\Omega}))^{d}, \mathbb{B}(\mathbf{P}(x))$ is uniformly bounded for all $x \in \bar{\Omega}$ and the boundedness of $L_{\mathbf{P}}$ follows from the Hölder's inequality. Thus the 
existence and uniqueness of a $H_{0}^{1}$-weak solution $(\mathbf{u}, \phi)$ follow directly from LaxMilgram.

It remains to show the $L^{p}$-isomorphism property of $L_{\mathbf{P}}$ for arbitrary $p \in$ $[2, \infty)$. Since $\mathbf{P}(\cdot)$ is uniformly continuous on $\bar{\Omega}$ and the coefficient tensor $\mathbb{B}(\cdot)$ is continuous in $\mathbf{P} \in \mathbb{R}^{d}$ due to Assumption $(\mathrm{C} 2)$, the coefficient tensor $\mathbb{B}(\mathbf{P}(\cdot))$ will also be uniformly continuous on $\bar{\Omega}$. On the other hand, the coerciveness of $L_{\mathbf{P}}$ implies that $L_{\mathbf{P}}$ is strongly elliptic in the sense of [13, Def. 3.36], see for instance [33. Thm. 4.6] for a proof. Then the claim follows from the elliptic regularity results given in 1 (when $\Omega$ is a $2 \mathrm{D}$-rectangle or a $3 \mathrm{D}$-cuboid) and [10] (when $\Omega$ has $C^{1}$-boundary) for strongly elliptic systems with uniformly continuous coefficients.

\subsection{Proof of Theorem 2.10}

In this section we give the proof of Theorem 2.10 In fact, we will show a slightly stronger version of Theorem 2.10, in the sense that we only require that $\mathbf{P}$ vanishes on $\operatorname{Sing}_{\Omega} \subset \Gamma_{\mathbf{P}}$.

Theorem 5.4. Let the Assumption (C2), Assumption 2.8 and (2.14) be satisfied. Let also $\Gamma_{\mathbf{u}}=\Gamma_{\phi}=\partial \Omega$. Then there exists some $p \in(3,6]$ such that $\mathrm{L}_{\mathbf{P}}$ is a topological isomorphism from $\left(W_{0}^{1, \mathfrak{p}}(\Omega)\right)^{4}$ to $\left(W^{-1, \mathfrak{p}}(\Omega)\right)^{4}$ for all $\mathfrak{p} \in[2, p]$ and for all $\mathbf{P} \in(C(\bar{\Omega}))^{3}$ with $\left.\mathbf{P}\right|_{\operatorname{Sing}_{\Omega}}=0$, where the set $\operatorname{Sing}_{\Omega}$ is defined by Assumption 2.8.

The rest of this section is devoted to the proof of Theorem 5.4. We firstly introduce the elliptic regularity result from 32 , stated in Theorem 5.5 below. Based on this result, we are then able to exploit perturbation arguments to complete the proof of Theorem 5.4.

Theorem 5.5 ([32, Thm. 4.3.5]). Let $\Omega$ be a polyhedral domain and $\lambda, \mu, \gamma$ be given positive constants. Assume also that for each vertex $x$ the corresponding cone $\mathcal{K}_{x}$ is a Lipschitz graph with edges, i.e. $\mathcal{K}_{x}$ has the representation $x_{3}>$ $\psi\left(x_{1}, x_{2}\right)$ in Cartesian coordinates with some Lipschitz function $\psi$. Define the differential operator $\mathrm{L}$ by

$$
\begin{aligned}
& \mathrm{L}:\left(H_{0}^{1}(\Omega)\right)^{4} \rightarrow\left(H^{-1}(\Omega)\right)^{4} \\
& \mathrm{~L}(\mathbf{u}, \phi)[\overline{\mathbf{u}}, \bar{\phi}]:=\int_{\Omega} \lambda \operatorname{tr}(\varepsilon(\mathbf{u})) \operatorname{tr}(\varepsilon(\overline{\mathbf{u}}))+2 \mu \varepsilon(\mathbf{u}): \varepsilon(\overline{\mathbf{u}})+\gamma \nabla \phi \cdot \nabla \bar{\phi} d x
\end{aligned}
$$

for $(\mathbf{u}, \phi),(\overline{\mathbf{u}}, \bar{\phi}) \in\left(H_{0}^{1}(\Omega)\right)^{4}$. Then there exists some $\tilde{p} \in(3, \infty)$ such that $\mathrm{L}$ is a topological isomorphism from $\left(W_{0}^{1, \mathfrak{p}}(\Omega)\right)^{4}$ to $\left(W^{-1, \mathfrak{p}}(\Omega)\right)^{4}$ for all $\mathfrak{p} \in[2, \tilde{p}]$.

Having all the preliminaries we are in the position to prove Theorem 5.4

Proof of Theorem 5.4. We follow a perturbation argument given by [27, Lem. A.18] to prove the statement. Consider a point $x \in \bar{\Omega}$. Let $\mathrm{L}_{\mathbf{P}}^{0}$ be the differential operator given by

$$
\mathrm{L}_{\mathbf{P}}^{0}(\mathbf{u}, \phi):=-\operatorname{Div}\left(\mathbb{B}\left(\mathbf{P}\left(x_{0}\right)\right)\left(\begin{array}{c}
\varepsilon(\mathbf{u}) \\
\nabla \phi
\end{array}\right)\right)
$$


with fixed constant coefficient tensor $\mathbb{B}\left(\mathbf{P}\left(x_{0}\right)\right)$ at $x_{0}=x$. Firstly we show that for each $x \in \bar{\Omega}$, there exist some neighborhood $U_{x}$ of $x$ and some $p_{x} \in(3,6]$ such that for all $\mathfrak{p} \in\left[2, p_{x}\right]$ we have

$$
\begin{aligned}
& \mathrm{L}_{\mathbf{P}}^{0} \text { is a topological isomorphism from } \\
& \left(W_{0}^{1, \mathfrak{p}}\left(U_{x} \cap \Omega\right)\right)^{4} \text { to }\left(W^{-1, \mathfrak{p}}\left(U_{x} \cap \Omega\right)\right)^{4} .
\end{aligned}
$$

We discuss different cases:

- If $x$ is in $\Omega$, then (5.3) follows from Theorem [5.3, since $U_{x} \subset \subset \Omega$ can be chosen as an open ball.

- If $x$ is on an open face, then by Definition 3.5, $x$ is on a smooth open twodimensional manifold. Thus we know that there exist some neighborhood $U_{x}$ of $x$ and a $C^{\infty}$-diffeomorphism $\iota$ such that $\iota$ maps $U_{x} \cap \Omega$ onto an open cube $\mathcal{C}$. According to the classical transformation arguments (see for instance [13, Thm. 4.14]), (5.3) is equivalent to the $W_{0}^{1, \mathfrak{p}} \leftrightarrow W^{-1, \mathfrak{p}}$ isomorphism property of a differential operator with suitable transformed coefficient tensor $\tilde{\mathbb{B}}$, which is still strongly elliptic, on $\mathcal{C}$. But the latter claim follows immediately from Theorem 5.3 .

- If $x \in \operatorname{Sing}_{\Omega}^{2} \cup \operatorname{Sing}_{\Omega}^{3}$, where $\operatorname{Sing}_{\Omega}^{2}, \operatorname{Sing}_{\Omega}^{3}$ are defined in Assumption 2.8, then by the definition of $\operatorname{Sing}_{\Omega}^{2}$ and $\operatorname{Sing}_{\Omega}^{3}$ we know that there exists a neighborhood $U_{x}$ such that the intersection $U_{x} \cap \Omega$ is $C^{\infty}$-diffeomorph to an open cube. Consequently, the claim follows from Theorem [5.3, by using the similar transformation arguments as the ones for the points on a face given previously.

- If $x \in \operatorname{Sing}_{\Omega}$, where $\operatorname{Sing}_{\Omega}$ is defined in Assumption 2.8, then due to (2.14), the operator $\mathrm{L}_{\mathbf{P}}^{0}$ reduces to the operator $\mathrm{L}$ defined in Theorem 5.5. In this case we simply take an arbitrary $U_{x}$ with $\bar{\Omega} \subset \subset U_{x}$ and the claim follows from Theorem 5.5.

This completes the proof of (5.3). Now let $\mathbf{P} \in(C(\bar{\Omega}))^{3}, \ell \in\left(W^{-1, \mathfrak{p}}(\Omega)\right)^{4}$ with $\mathfrak{p} \in[2,6]$ and let $(\mathbf{u}, \phi)$ be the unique $H_{0}^{1}$-solution of

$$
\mathrm{L}_{\mathbf{P}}(\mathbf{u}, \phi)=\ell \quad \text { in } \Omega,
$$

whose existence and uniqueness are guaranteed by Lax-Milgram. Consider a point $x \in \bar{\Omega}$. Let $\eta: \mathbb{R}^{3} \rightarrow \mathbb{R}$ be a real valued smooth function such that $\operatorname{supp}(\eta) \subset U_{x}$, where $U_{x}$ is as defined in (5.3). We also assume that $\mathfrak{p} \in$ $\left[2, p_{x}\right]$ with $p_{x}$ given by (5.3). Define $W:=(\mathbf{u}, \phi), V:=(\overline{\mathbf{u}}, \bar{\phi})$ and rewrite $\mathrm{L}_{\mathbf{P}}(\mathbf{u}, \phi)[\overline{\mathbf{u}}, \bar{\phi}]$ a⿺

$$
\mathrm{L}_{\mathbf{P}}(\mathbf{u}, \phi)[\overline{\mathbf{u}}, \bar{\phi}]=\int_{\Omega} \mathbb{A}_{i j}^{\alpha \beta} \partial_{\beta} W^{j} \partial_{\alpha} V^{i} d x
$$

with coefficient tensor $\mathbb{A}$. For $V \in\left(C_{0}^{\infty}\left(U_{x} \cap \Omega\right)\right)^{4}$ we obtain that

$$
\int_{U_{x} \cap \Omega} \mathbb{A}_{i j}^{\alpha \beta} \partial_{\beta}(\eta W)^{j} \partial_{\alpha} V^{i} d x
$$

\footnotetext{
${ }^{3}$ We use here and below the Einstein summation notation.
} 


$$
\begin{aligned}
& =\int_{U_{x} \cap \Omega} \mathbb{A}_{i j}^{\alpha \beta}\left(\partial_{\beta} \eta\right) W^{j} \partial_{\alpha} V^{i}+\mathbb{A}_{i j}^{\alpha \beta} \eta \partial_{\beta} W^{j} \partial_{\alpha} V^{i} d x \\
& =\int_{U_{x} \cap \Omega} \mathbb{A}_{i j}^{\alpha \beta}\left(\partial_{\beta} \eta\right) W^{j} \partial_{\alpha} V^{i}+\mathbb{A}_{i j}^{\alpha \beta} \partial_{\beta} W^{j} \partial_{\alpha}(\eta V)^{i}+\left(-\mathbb{A}_{i j}^{\alpha \beta} \partial_{\beta} W^{j}\left(\partial_{\alpha} \eta\right) V^{i}\right) d x \\
& =: \ell_{a}(V)+\ell(\eta V)+\ell_{b}(V)=: \tilde{\ell}(V),
\end{aligned}
$$

where $\ell(\eta V)$ is understood as the evaluation of $\ell$ at the extension of $\eta V$ on whole $\Omega$ such that the extension is zero on $\Omega \backslash U_{x}$. From fundamental calculus one obtains that

$$
\begin{aligned}
& q \in[2,6] \Rightarrow 1-\frac{3}{2} \geq 0-\frac{3}{q}, \\
& q \in[2,6] \Rightarrow 0-\frac{3}{2} \geq-1-\frac{3}{q} .
\end{aligned}
$$

Consequently, using Sobolev embedding one verifies that for $q \in[2,6], \operatorname{Div}(\mathbf{u}, \phi)$ and $\nabla(\mathbf{u}, \phi)$ are of class $W^{-1, q}$ if $(\mathbf{u}, \phi)$ is of class $H^{1}$. Thus $\ell_{a}, \ell_{b}$ are of class $W^{-1, q}$ for $q \in[2,6]$. Moreover, we infer that $\tilde{\ell}$ is of class $W^{-1, q}$ if and only if $\ell$ is of class $W^{-1, q}$ for $q \in[2,6]$. Since $\mathbf{P}$ is uniformly continuous on $\bar{\Omega}$, the coefficient tensor $\mathbb{A}$ is also uniformly continuous and consequently uniformly bounded on $\bar{\Omega}$. Letting $q=\mathfrak{p}$ it follows from Hölder's inequality that

$$
\begin{aligned}
& \|\tilde{\ell}\|_{W^{-1, \mathfrak{p}}\left(U_{x} \cap \Omega\right)} \\
\leq & C\left(\|\ell\|_{W^{-1, \mathfrak{p}}(\Omega)}+\|(\mathbf{u}, \phi)\|_{L^{\mathfrak{p}}(\Omega)}+\|\nabla(\mathbf{u}, \phi)\|_{L^{2}(\Omega)}\right) .
\end{aligned}
$$

Additionally we obtain that $\eta(\mathbf{u}, \phi)$ is the $H_{0}^{1}$-solution of

$$
\mathrm{L}_{\mathbf{P}}(\eta(\mathbf{u}, \phi))=\tilde{\ell} \quad \text { in } U_{x} \cap \Omega .
$$

For $r_{x}>0$ with $\bar{B}_{r_{x}}(x) \subset U_{x}$ define the tensor $\mathbb{B}(\mathbf{P})^{*}$ on $U_{x} \cap \Omega$ by

$$
\mathbb{B}(\mathbf{P})^{*}(y):= \begin{cases}\mathbb{B}(\mathbf{P}(x)), & y \in\left(U_{x} \cap \Omega\right) \backslash B_{r_{x}}(x), \\ \mathbb{B}(\mathbf{P}(y)), & y \in\left(U_{x} \cap \Omega\right) \cap B_{r_{x}}(x) .\end{cases}
$$

Denote by $\mathrm{L}_{\mathbf{P}}^{*}$ the differential operator given by

$$
\mathrm{L}_{\mathbf{P}}^{*}(\mathbf{u}, \phi):=-\operatorname{Div}\left(\mathbb{B}(\mathbf{P})^{*}\left(\begin{array}{c}
\varepsilon(\mathbf{u}) \\
\nabla \phi
\end{array}\right)\right) .
$$

Using the fact that the coefficient tensor $\mathbb{B}$ is continuous on $\mathbb{R}^{3}$ from Assumption (C2) and that $\mathbf{P}$ is uniformly continuous on the whole $\bar{\Omega}$ we deduce that $\mathbb{B}(\mathbf{P}(\cdot))$ is uniformly continuous on $\bar{\Omega}$. This, together with Hölder's inequality, implies that there exists a sufficiently small $r_{x}>0$ such that

$$
\begin{aligned}
& \left\|\mathrm{L}_{\mathbf{P}}^{*}-\mathrm{L}_{\mathbf{P}}^{0}\right\|_{L\left(W_{0}^{1, \mathfrak{p}}\left(U_{x} \cap \Omega\right), W^{-1, \mathfrak{p}}\left(U_{x} \cap \Omega\right)\right)}\left\|\left(\mathrm{L}_{\mathbf{P}}^{0}\right)^{-1}\right\|_{L\left(W^{-1, \mathfrak{p}}\left(U_{x} \cap \Omega\right), W_{0}^{1, \mathfrak{p}}\left(U_{x} \cap \Omega\right)\right)} \\
\leq & C\|\mathbb{B}(\mathbf{P}(\cdot))-\mathbb{B}(\mathbf{P}(x))\|_{L^{\infty}\left(B_{r_{x}}(x) \cap \Omega\right)}\left\|\left(\mathrm{L}_{\mathbf{P}}^{0}\right)^{-1}\right\|_{L\left(W^{-1, \mathfrak{p}}\left(U_{x} \cap \Omega\right), W_{0}^{1, \mathfrak{p}}\left(U_{x} \cap \Omega\right)\right)} \\
< & 1 .
\end{aligned}
$$

Combining with the small perturbation theorem [24, Chap. 4, Thm. 1.16] and (5.3) we infer that $\mathrm{L}_{\mathbf{P}}^{*}$ is a topological isomorphism from $\left(W_{0}^{1, \mathfrak{p}}\left(U_{x} \cap \Omega\right)\right)^{4}$ to 
$\left(W^{-1, \mathfrak{p}}\left(U_{x} \cap \Omega\right)\right)^{4}$ for all $\mathfrak{p} \in\left[2, p_{x}\right]$. If we additionally let $\operatorname{supp}(\eta) \subset B_{r_{x}(x)}$, then we deduce that $\eta(\mathbf{u}, \phi)$ is the $H_{0}^{1}$-solution of

$$
\mathrm{L}_{\mathbf{P}}^{*}(\eta(\mathbf{u}, \phi))=\tilde{\ell} \quad \text { in } U_{x} \cap \Omega .
$$

This shows that $\eta(\mathbf{u}, \phi)$ is of class $W^{1, \mathfrak{p}}$ on $U_{x} \cap \Omega$ for all $\mathfrak{p} \in\left[2, p_{x}\right]$.

Finally, we use a covering argument to finish the desired proof. Let $U_{x}, p_{x}$ be as defined in (5.3) and $r_{x}$ the small number constructed in the previous step. Since $\Omega$ is a bounded domain in $\mathbb{R}^{3}$, we can find some $k \in \mathbb{N}$ such that $\bar{\Omega} \subset \cup_{i=1}^{k} B_{r_{x_{i}}}\left(x_{i}\right)$ with $x_{i} \in \bar{\Omega}$. Define

$$
p:=\min \left\{p_{x_{1}}, \ldots, p_{x_{k}}\right\} \in(3,6] .
$$

At this point we emphasize that $p$ can be chosen in a (possibly non-optimal) way that $p$ is independent of the choice of $\mathbf{P}$. Indeed, along the proof of (5.3) it is easy to see that one can always set $p_{x} \equiv \min \{\tilde{p}, 6\}$, where $\tilde{p}$ is given by Theorem 5.5 which is independent of $\mathbf{P}$. Now let $\mathfrak{p} \in[2, p]$. Let $\left\{\eta_{i}\right\}_{i=1}^{k}$ be a partition of unity subordinated to the balls $\left\{B_{r_{x_{i}}}\left(x_{i}\right)\right\}_{i=1}^{k}$. Then there is a corresponding $\tilde{\ell}_{i}$ of class $W^{-1, \mathfrak{p}}$, similarly defined as in (5.5), such that $\eta_{i}(\mathbf{u}, \phi)$ is the $H_{0}^{1}$-solution of

$$
\mathrm{L}_{\mathbf{P}}^{*}\left(\eta_{i}(\mathbf{u}, \phi)\right)=\tilde{\ell}_{i} \quad \text { in } U_{x_{i}} \cap \Omega .
$$

We obtain that

$$
\begin{aligned}
& \|(\mathbf{u}, \phi)\|_{W_{0}^{1, \mathfrak{p}}(\Omega)} \\
\leq & \sum_{i=1}^{k}\left\|\eta_{i}(\mathbf{u}, \phi)\right\|_{W_{0}^{1, \mathfrak{p}}\left(U_{x_{i}} \cap \Omega\right)} \\
\leq & C \sum_{i=1}^{k}\left\|\tilde{\ell}_{i}\right\|_{W^{-1, \mathfrak{p}}\left(U_{x_{i}} \cap \Omega\right)} \\
\leq & C\left(\|\ell\|_{W^{-1, \mathfrak{p}(\Omega)}}+\|(\mathbf{u}, \phi)\|_{L^{\mathfrak{p}}(\Omega)}+\|\nabla(\mathbf{u}, \phi)\|_{L^{2}(\Omega)}\right)<\infty .
\end{aligned}
$$

This completes the proof of Theorem 5.4

\section{Acknowledgments}

This research was supported by the doctoral program of the University of Kassel. The author wants to extend his sincere thanks to Prof. Dr. Dorothee Knees for her meticulous care and great support during the author's doctoral study at the University of Kassel. The author also wants to thank the anonymous referees for their valuable comments and suggestions. Special thanks also to a referee for suggesting the replacement of the volume-preserving condition in an older version of the manuscript to the more general Ahlfors-David-regular condition, which greatly improves the applicability of the results in the present paper.

\section{References}

[1] Acquistapace, P. On BMO regularity for linear elliptic systems. Ann. Mat. Pura Appl. (4) 161 (1992), 231-269. 
[2] Ahlfors, L. Zur Theorie der Überlagerungsflächen. Acta Math. 65, 1 (1935), 157-194.

[3] Amann, H. Linear parabolic problems involving measures. RACSAM. Rev. R. Acad. Cienc. Exactas Fís. Nat. Ser. A Mat. 95, 1 (2001), 85-119.

[4] Auscher, P., Badr, N., Haller-Dintelmann, R., and Rehberg, J. The square root problem for second-order, divergence form operators with mixed boundary conditions on $L^{p}$. J. Evol. Equ. 15, 1 (2015), 165-208.

[5] Bartic, A. T., Wouters, D. J., Maes, H. E., Rickes, J. T., And WASER, R. M. Preisach model for the simulation of ferroelectric capacitors. Journal of Applied Physics 89, 6 (mar 2001), 3420-3425.

[6] Bergh, J., AND LÖFström, J. Interpolation spaces. An introduction. Grundlehren der Mathematischen Wissenschaften, No. 223. SpringerVerlag, Berlin-New York, 1976.

[7] Clément, P., And Li, S. Abstract parabolic quasilinear equations and application to a groundwater flow problem. Adv. Math. Sci. Appl. 3, Special Issue (1993/94), 17-32.

[8] Cross, L. E., And Newnham, R. E. History of ferroelectrics, ceramics and civilization. High-technology ceramics - past, present and future, vol. 3. American Ceramic Society, 1987, pp. 289-305.

[9] David, G. Opérateurs intégraux singuliers sur certaines courbes du plan complexe. Ann. Sci. École Norm. Sup. (4) 17, 1 (1984), 157-189.

[10] Dolzmann, G., AND MÜLler, S. Estimates for Green's matrices of elliptic systems by $L^{p}$ theory. Manuscripta Math. 88, 2 (1995), 261-273.

[11] Dore, G. $L^{p}$ regularity for abstract differential equations. In Functional analysis and related topics, 1991 (Kyoto), vol. 1540 of Lecture Notes in Math. Springer, Berlin, 1993, pp. 25-38.

[12] Egert, M. $L^{p}$-estimates for the square root of elliptic systems with mixed boundary conditions. J. Differential Equations 265, 4 (2018), 1279-1323.

[13] Giaquinta, M., and Martinazzi, L. An introduction to the regularity theory for elliptic systems, harmonic maps and minimal graphs, second ed., vol. 11 of Appunti. Scuola Normale Superiore di Pisa (Nuova Serie) [Lecture Notes. Scuola Normale Superiore di Pisa (New Series)]. Edizioni della Normale, Pisa, 2012.

[14] Griepentrog, J. A., Gröger, K., Kaiser, H.-C., And Rehberg, J. Interpolation for function spaces related to mixed boundary value problems. Math. Nachr. 241 (2002), 110-120.

[15] Grisvard, P. Elliptic problems in nonsmooth domains, vol. 24 of Monographs and Studies in Mathematics. Pitman (Advanced Publishing Program), Boston, MA, 1985. 
[16] Gröger, K., AND Rehberg, J. Resolvent estimates in $W^{-1, p}$ for second order elliptic differential operators in case of mixed boundary conditions. Math. Ann. 285, 1 (1989), 105-113.

[17] Haertling, G. H. Ferroelectric ceramics: History and technology. Journal of the American Ceramic Society 82, 4 (apr 1999), 797-818.

[18] Haller-Dintelmann, R., Jonsson, A., Knees, D., And Rehberg, J. Elliptic and parabolic regularity for second-order divergence operators with mixed boundary conditions. Math. Methods Appl. Sci. 39, 17 (2016), $5007-5026$.

[19] Haller-Dintelmann, R., And Rehberg, J. Maximal parabolic regularity for divergence operators including mixed boundary conditions. $J$. Differential Equations 247, 5 (2009), 1354-1396.

[20] Huber, J. E., Fleck, N. A., Landis, C. M., and McMeeking, R. M. A constitutive model for ferroelectric polycrystals. J. Mech. Phys. Solids 47, 8 (1999), 1663-1697.

[21] Jonsson, A., And Wallin, H. Function spaces on subsets of $\mathbf{R}^{n}$, vol. 2 . 1984.

[22] Kaltenbacher, M., And Kaltenbacher, B. Modeling and numerical simulation of ferroelectric material behavior using hysteresis operators. In Ferroelectrics - Characterization and Modeling. InTech, aug 2011.

[23] Kamlah, M. Ferroelectric and ferroelastic piezoceramics - modeling of electromechanical hysteresis phenomena. Continuum Mechanics and Thermodynamics 13, 4 (Aug 2001), 219-268.

[24] Kato, T. Perturbation theory for linear operators. Classics in Mathematics. Springer-Verlag, Berlin, 1995. Reprint of the 1980 edition.

[25] KneEs, D. Convergence analysis of time-discretisation schemes for rateindependent systems. ESAIM: Control, Optimisation and Calculus of Variations 25 (2019), 65.

[26] Kozlov, V. A., Maz'ya, V. G., and Rossmann, J. Spectral problems associated with corner singularities of solutions to elliptic equations, vol. 85 of Mathematical Surveys and Monographs. American Mathematical Society, Providence, RI, 2001.

[27] Krumbiegel, K., And Rehberg, J. Second order sufficient optimality conditions for parabolic optimal control problems with pointwise state constraints. SIAM J. Control Optim. 51, 1 (2013), 304-331.

[28] Kutter, M., And SÄNDIG, A.-M. Modeling of ferroelectric hysteresis as variational inequality. GAMM-Mitt. 34, 1 (2011), 84-89.

[29] Luo, Y. Existence and Regularity Results of a Ferroelectric Phase-Field Model. Ph.D. thesis, University of Kassel, 2019. 
[30] Marton, P., Rychetsky, I., And Hlinka, J. Domain walls of ferroelectric $\mathrm{BaTiO}_{3}$ within the Ginzburg-Landau-Devonshire phenomenological model. Phys. Rev. B 81 (Apr 2010), 144125.

[31] Mattila, P., Melnikov, M. S., And Verdera, J. The Cauchy integral, analytic capacity, and uniform rectifiability. Ann. of Math. (2) 144, 1 (1996), 127-136.

[32] Maz'ya, V., AND Rossmann, J. Elliptic equations in polyhedral domains, vol. 162 of Mathematical Surveys and Monographs. American Mathematical Society, Providence, RI, 2010.

[33] MCLean, W. Strongly elliptic systems and boundary integral equations. Cambridge University Press, Cambridge, 2000.

[34] Meinlschmidt, H., Meyer, C., And Rehberg, J. Optimal control of the thermistor problem in three spatial dimensions, Part 1: Existence of optimal solutions. SIAM J. Control Optim. 55, 5 (2017), 2876-2904.

[35] Miene, C., And Rosato, D. A rate-dependent incremental variational formulation of ferroelectricity. Internat. J. Engrg. Sci. 49, 6 (2011), 466496.

[36] Mielke, A., And RoubíčEK, T. Rate-independent systems, vol. 193 of Applied Mathematical Sciences. Springer, New York, 2015. Theory and application.

[37] Mielke, A., And Timofte, A. M. An energetic material model for time-dependent ferroelectric behaviour: existence and uniqueness. Math. Methods Appl. Sci. 29, 12 (2006), 1393-1410.

[38] Pechstein, A. S., Meindlhumer, M., And Humer, A. The polarization process of ferroelectric materials in the framework of variational inequalities. ZAMM Z. Angew. Math. Mech. 100, 6 (2020), 201900329, 18.

[39] PRüss, J. Maximal regularity for evolution equations in $L_{p}$-spaces. Conf. Semin. Mat. Univ. Bari, 285 (2002), 1-39 (2003).

[40] Schrade, D., Keip, M.-A., Thai, H., Schröder, J., Svendsen, B., Müller, R., AND Gross, D. Coordinate-invariant phase field modeling of ferro-electrics, part I: Model formulation and single-crystal simulations. GAMM-Mitt. 38, 1 (2015), 102-114.

[41] Schrade, D., Müller, R., And Gross, D. On the physical interpretation of material parameters in phase field models for ferroelectrics. Archive of Applied Mechanics 83, 10 (may 2013), 1393-1413.

[42] SchröDER, J., AND Romanowski, H. A thermodynamically consistent mesoscopic model for transversely isotropic ferroelectric ceramics in a coordinate-invariant setting. Archive of Applied Mechanics 74, 11 (Nov 2005), 863-877.

[43] Su, Y., AND Landis, C. M. Continuum thermodynamics of ferroelectric domain evolution: theory, finite element implementation, and application to domain wall pinning. J. Mech. Phys. Solids 55, 2 (2007), 280-305. 
[44] Triebel, H. Interpolation theory, function spaces, differential operators, vol. 18 of North-Holland Mathematical Library. North-Holland Publishing Co., Amsterdam-New York, 1978.

[45] Werner, K. Ferroelectrics and Antiferroelectrics. Academic Press, Amsterdam, Boston, 1964. 